\title{
Using intracerebral microdialysis to probe the efficacy of repurposed drugs in Alzheimer's disease pathology
}

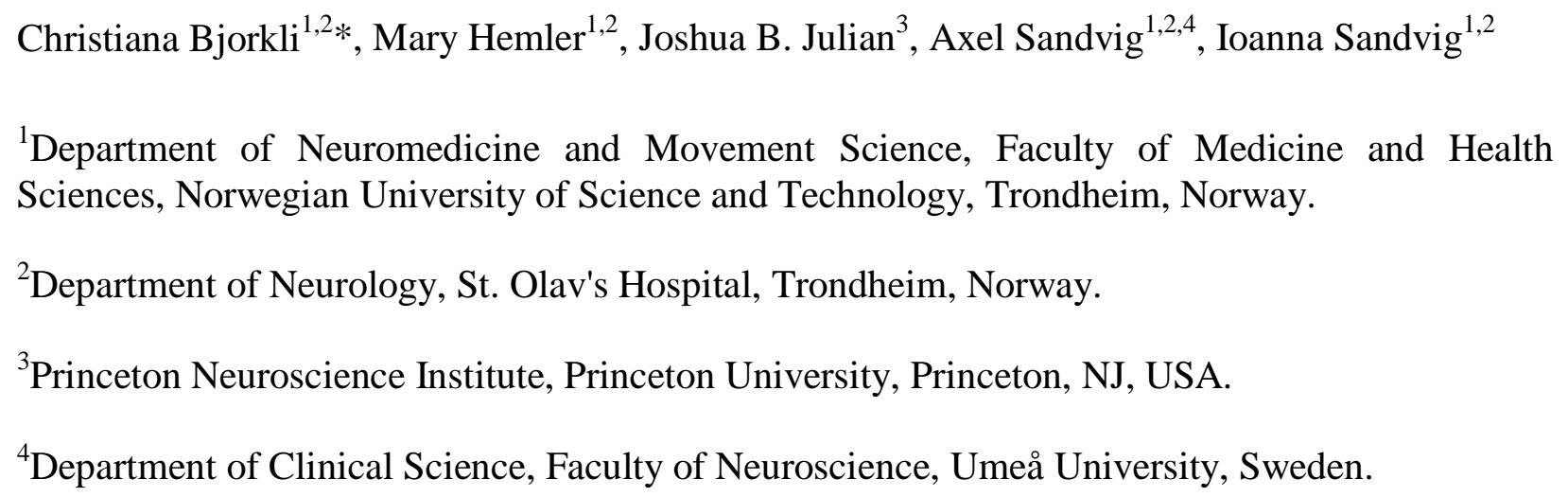

One Sentence Summary: Here we circumvented the blood-brain barrier for drug delivery aimed at attenuating $\mathrm{AD}$ neuropathology.

Abstract: All disease-targeting drug trials completed to date have fallen short of meeting the clinical endpoint of significantly slowing cognitive decline in Alzheimer's disease patients. Even the recently approved drug Aducanumab, has proven effective in removing amyloid- $\beta$, but does not reduce cognitive decline. This emphasizes the urgent need for novel therapeutic approaches that could reduce several AD neuropathologies simultaneously, eventually leading to improved cognitive performance. To validate whether our mouse model replicates AD neuropathology as observed in patients, we characterized the $3 \times \mathrm{Tg}$ AD mouse model to avoid premature translation of successful results. In this study we have repurposed two FDA-approved drugs, Fasudil and Lonafarnib, targeting the Wnt signaling and endosomal-lysosomal pathway respectively, to test their potential to attenuate $\mathrm{AD}$ pathology. Using intracerebral microdialysis, we simultaneously infused these disease-targeting drugs between 1-2 weeks, separately and also in combination, while collecting cerebrospinal fluid. We found that Fasudil reduces intracellular amyloid- $\beta$ in young, and amyloid plaques in old animals, and overall cerebrospinal fluid amyloid- $\beta$. Lonafarnib reduces tau neuropathology and cerebrospinal fluid tau biomarkers in young and old animals. Co-infusion of both drugs was more effective in reducing intracellular amyloid- $\beta$ than either drug alone, and appeared to improve contextual memory performance. However, an unexpected finding was that Lonafarnib treatment increased amyloid plaque size, suggesting that activating the endosomal-lysosomal system may inadvertently increase amyloid- $\beta$ pathology if administered too late in the AD continuum. Taken together, these findings lend support to the application of repurposed drugs to attenuate $\mathrm{AD}$ neuropathology at various therapeutic time 39 windows. 
Alzheimer's disease (AD) is the leading cause of dementia in older adults. Symptoms feature progressive neurodegeneration, followed by impairments in memory, cognitive and visuospatial function (1). Brain pathology in AD patients is characterized by abiotrophic neuronal cell death, vascular abnormalities, extracellular deposits of amyloid- $\beta$ (A $\beta)$, and neurofibrillary tangles (NFTs) (1). In most cases, the cause of AD is not known. However, rare inherited versions of $\mathrm{AD}$ are often caused by mutations that result in increased production of $\mathrm{A} \beta(2,3)$ and lead to familial forms of the disease. Both amyloid plaques and NFTs are believed to interfere with cytoskeletal integrity as well as disrupting axonal transport, and the healthy function of synapses and neurons (4). Research within the field has primarily focused on late-stage disease pathology and a central reason for recent failures in clinical trials in $\mathrm{AD}$ may thus be the timing of intervention, as most clinical trials begin at advanced stages of the disease, after irreversible neuronal death has already occurred $(5,6)$. It is therefore our view that research efforts need to also focus on earlier stages of $\mathrm{AD}$, when therapeutic interventions would likely be more effective.

Cerebrospinal fluid (CSF) levels of $A \beta$ and tau protein demonstrate high accuracy for identifying incipient $\mathrm{AD}$ as well as predicting the development and rate of cognitive decline in the clinic $(4$, 7-9). Lower concentrations of CSF A $\beta 42$ and higher concentrations of total tau (t-tau) protein have been used to distinguish $\mathrm{AD}$ patients from cognitively normal age-matched controls and to predict the transition from mild cognitive impairment (MCI) to $\mathrm{AD}(4,7-9)$. Studies indicate that abnormal levels of CSF A $\beta$ manifest several years prior to the appearance of subjective memory complaints, making CSF $\mathrm{A} \beta$ the current earliest biomarker for $\mathrm{AD}(10,11)$. In the early phases of $\mathrm{AD}$, there is evidence that CSF $\mathrm{A} \beta 42$ levels significantly increase, initially reflecting the rate of $\mathrm{A} \beta 42$ production in the brain (12). However, as the disease progresses, AD patients have diminished CSF A $\beta 42$ levels due to a reduction of its clearance and the formation of amyloid plaques in the brain parenchyma (13). Recent studies support the hypothesis that the accumulation of $A \beta$ peptides within the brain arises from an imbalance in the production and clearance of $A \beta$, and the ability to clear $A \beta$ diminishes with age (14). Levels of CSF tau serve as an indication of its pathogenesis in the cerebral cortex; both t- and phosphorylated (p)-tau levels increase during progression of $\mathrm{AD}$ and correlate with cognitive decline (15). It is believed that the decrease in CSF A $\beta 42$ reflects its aggregation and deposition in the brain parenchyma, whereas the increase in CSF tau reflects its extracellular release after neuronal degeneration and NFT formation (9). Thus, these biomarkers are considered to be directly linked to the molecular pathogenesis of AD. Since memory impairment manifests in many other diseases in addition to $\mathrm{AD}$, biomarkers are often left as the sole criteria for accurate diagnosis.

Over the past 25 years, there has been an abundance of research on the development of therapies aimed at delaying or halting the progression of $\mathrm{AD}(16,17)$. Despite this research, diseasetargeting drugs have repeatedly failed to demonstrate success in clinical trials (18). A $\beta$ immunotherapy in mouse models of $\mathrm{AD}$ has been shown to reduce intracellular $\mathrm{A} \beta$ and amyloid plaque accumulation and to lead to a greater clearance of p-tau pathology; however, aggregates of hyperphosphorylated tau remained unaffected by $\mathrm{A} \beta$ antibody immunization treatment (19). This suggests that $\mathrm{A} \beta$ targeting therapies may be more effective at the preclinical stages of $\mathrm{AD}$, but once cognitive decline appears in tandem with tau pathology, tau-targeting drugs may be necessary (20). Moreover, the most commonly used $\mathrm{AD}$ animal models are mice that overexpress human genes associated with familial AD that result in the formation of amyloid plaques. However, $\mathrm{AD}$ is defined by the presence and interplay of both amyloid plaques and 87 NFT pathology (21). There has thus been a poor track record of success in AD clinical trials, and 
this is in part due to the premature translation of successful results in animal models that mirror only limited aspects of $\mathrm{AD}$ pathology in patients. Here we have used the $3 \mathrm{xTg} \mathrm{AD}$ mouse model (22) which harbors human AD-related genetic mutations resulting in amyloid plaques and NFTs.

In this study we aimed to target the blood-CSF barrier, formed by the epithelium of the choroid plexuses, for delivery of drugs attenuating AD pathology in the brain parenchyma. First, we repurposed Fasudil, which is used to treat cerebral vasospasm. Fasudil works by targeting Rhoassociated protein kinase (ROCK) in the Wnt-PCP signaling pathway (Fig. 1), effectively derailing the synaptotoxic cascade of $A \beta$ production. Previous research has demonstrated that ROCK kinases can induce the processing of amyloid precursor protein (APP) to the toxic A $\beta 42$ peptide and that this can be prevented by ROCK inhibition (23). Wnt-PCP synaptic signaling is triggered from the increased presence of $\mathrm{A} \beta$ protein, and increased expression of Dickkopf WNT Signaling Pathway Inhibitor 1 (Dkk1) has been shown in post-mortem AD brains and in animal

100 models of $A \beta$ pathology $(24,25)$. Moreover, activating autophagy (Fig. 2$)$ has increasingly been

101 considered as a potential therapeutic treatment for $\mathrm{AD}$, with animal and cellular models showing

102 that autophagy activators can reduce the levels of misfolded and aggregation proteins, prevent the spread of tau, and reduce neuronal loss (26-28). The second drug we repurposed is

104 Lonafarnib, originally a cancer drug and an autophagic activator, which has been found to direct abnormal tau protein into lysosomes, and thereby remove tau before it can form NFTs (26).

Current $\mathrm{AD}$ drug development is limited by the blood-brain barrier (BBB), as more than $98 \%$ of all molecular drugs do not penetrate it. Although several lines of research have been developed to investigate transport functions across the $\mathrm{BBB}$, in vivo brain microdialysis seems to be one of the most suitable techniques for characterizing the influx and efflux transport functions across the barrier under physiological and pathological conditions (29). We predicted that drugs infused into the lateral ventricle $\mathrm{f} 3 \mathrm{xTg} \mathrm{AD}$ mice would reach deep cerebral structures, such as the 113 hippocampus (HPC), and that such chronic drug delivery would reduce CSF sink action towards 114 drugs (30). We additionally hypothesized that the earlier the biochemical events leading to the deposition of amyloid plaques and NFTs were targeted, the more effective treatment would be and that drug treatment could rescue contextual memory deficits in mice. 
bioRxiv preprint doi: https://doi.org/10.1101/2022.01.14.476357; this version posted January 14, 2022. The copyright holder for this preprint (which was not certified by peer review) is the author/funder. All rights reserved. No reuse allowed without permission.

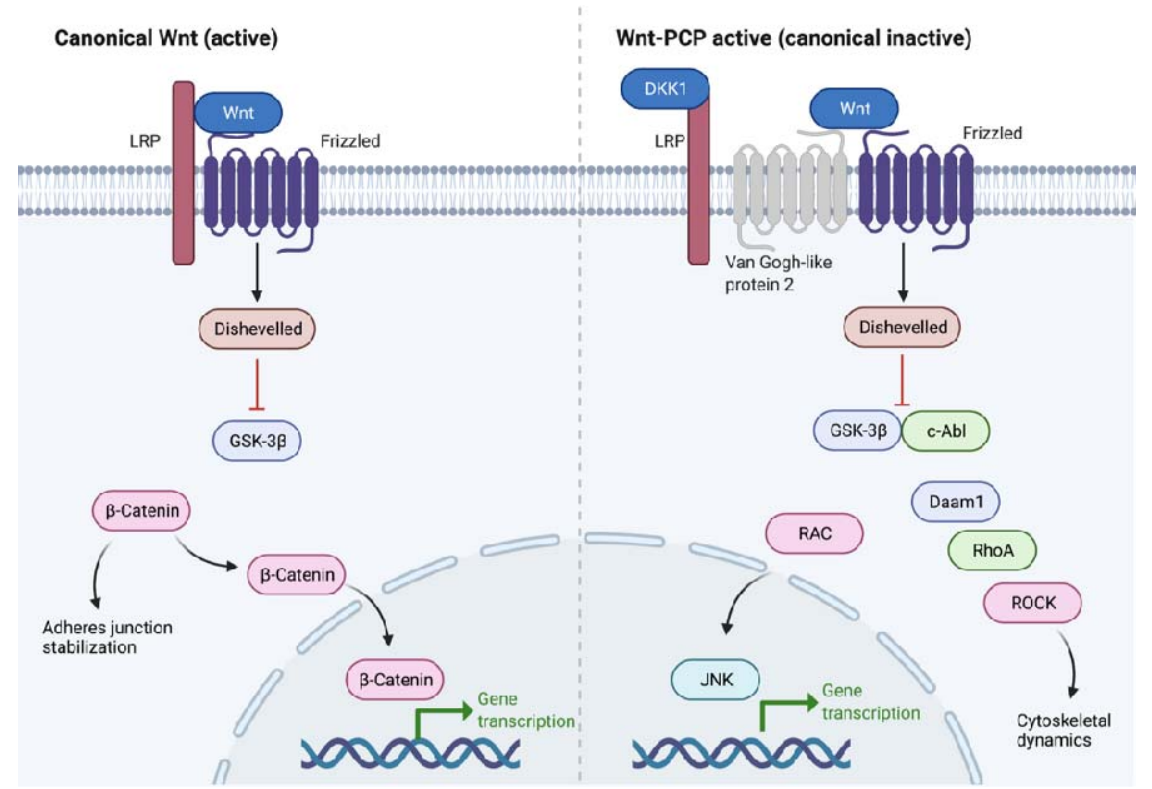

Figure 1. Schematic of the canonical Wnt and Wnt-PCP pathways. A $\beta$ has been shown to activate the Wnt-PCP pathway through the ability of $A \beta$ to induce Dkk1. Dkk1 then prevents the binding interaction between LRP6 and frizzled, activating WntPCP signaling and blocking canonical Wnt- $\beta$-catenin activity. In the Wnt-PCP pathway, the two arms diverge below disheveled, acting via Daam/RhoA/ROCK to regulate cytoskeletal dynamics and JNK/c-Jun to regulate gene transcription. Figured adapted from (24). Abbreviations; Daam1: disheveled associated activator of morphogenesis 1; Dkk1: Dickkopf-1; GSK-3ß: glycogen synthase kinase-3 $\beta$; JNK: c-Jun N-terminal kinase; LRP: low-density lipoprotein receptor-related protein; PCP: planar cell polarity; RhoA: Ras homolog family member A; ROCK: Rho-associated coiled-coil containing protein kinase; Wnt: Winglessrelated integration site. Figure created with biorender.com.

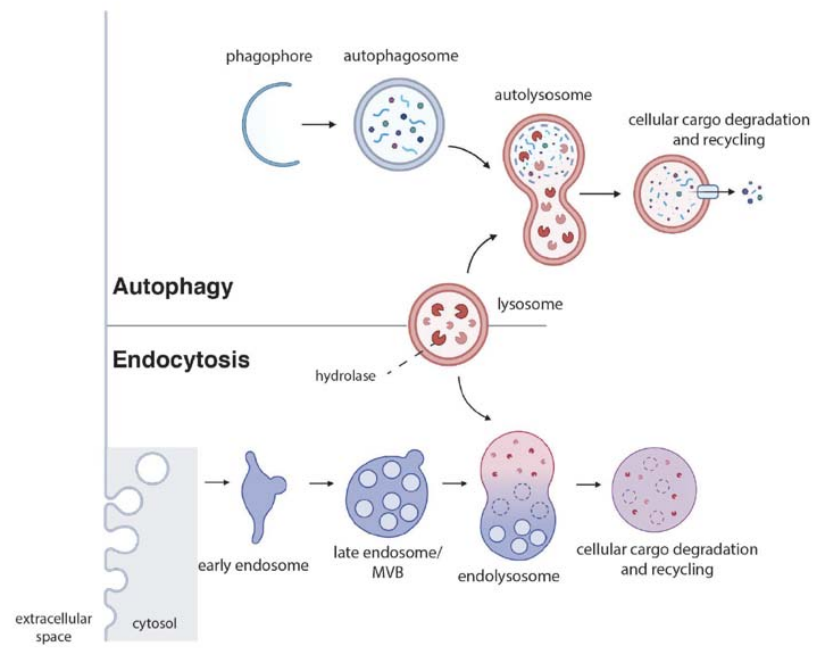

Figure 2. Overview of the autophagic and endocytic pathways. In macroautophagy, the phagophore encloses a portion of cytoplasm that contains organelles and large proteins destined for degradation, forming an autophagosome. The autophagosome then fuses with a lysosome, forming an autolysosome. Lysosomal hydrolases then degrade the internalized material and recycle components back to the plasma membrane. Endocytosis first involves internalization of extracellular material and plasma membrane, which are then delivered to the early endosome. After progressive maturation of the early endosome into a late endosome (also referred to as a multivesicular body), the late endosome fuses with a lysosome. As in autophagy, cellular cargo in the lysosome is then degraded via acid hydrolases. Therefore, both the autophagic and the endocytic pathways converge on the lysosome. Figure adapted from (31) and created with biorender.com. 


\section{The 3xTg AD mouse model displays Alzheimer's disease-related neuropathology}

139 To validate whether our mouse model replicated AD neuropathology as observed in patients, and 140 to assess genetic drift in our own colony (32), we first characterized the 3xTg AD mouse model. 141 In the present characterization study intracellular $A \beta$ immunoreactivity is observed in frontal and 142 sensory areas, the HPC, and parts of the cerebellum as early as 1 month of age (Fig. 3A, B). Regarding extracellular amyloid plaque accumulation, these are observed first at 13 months of age in the dorsal subiculum (dSub; Fig. 3A, B). The observed amyloid plaques are immunoreactive to both McSA1 (A $\beta 38-42)$ and OC (fibrillar $A \beta$ ) antibodies, suggesting that these plaques consist of fibrillar $A \beta 38-42$. In addition, reactive microglia (Iba1 and TREM2 immunolabelling) surround deposited plaques in this mouse model (Fig. 3C, D). At 1 month of age, there is $\mathrm{A} \beta 38-42$ immunoreactivity in large parts of the cortex, but not $\mathrm{A} \beta 42$ immunoreactivity. Oligomeric $A \beta$ is not present at 1 month of age (Supplementary Fig. 1), whereas fibrillar $\mathrm{A} \beta(\mathrm{OC})$ is present in the frontal cortex. Moreover, amyloid plaques immunoreactive to A $\beta 38-42$ are first apparent at 13 months of age in dSub, whereas plaques immunoreactive to $A \beta 42$ are first apparent at 15 months of age in dSub (Fig. 3B). MC1 immunoreactivity (AD-specific conformation modification of tau) is found within OC-positive amyloid plaques in the dSub of 3xTg AD mice (Fig. 3E).

In the current study, intracellular tau immunoreactive to HT7 (endogenous microtubuleassociated protein tau [MAPT]) is present in frontal and sensory areas, and the HPC as early as 1 month of age (Fig. 3F). The HT7 immunoreactivity appears to be less pronounced in 13-monthold mice compared to 1 month old mice, suggesting that tau levels decrease in later stages of AD. Regarding pathological tau (AT8 antibody) immunoreactivity in 13-month-old 3xTg AD mice, there is immunolabelling in CA1 and caudal subiculum at this age, but not immunolabelling in dSub (Fig. 3G). At 13 months of age, caudal subiculum appears to have strongest immunolabelling, and is therefore the first region involved in tau pathology in this model, at least in terms of hyperphosphorylated tau. There are NFTs present in pyramidal CA1 neurons at 18 months of age, and the brain region with the most prominent AT8 immunoreactivity is the caudal subiculum at 17 months of age. Gallyas-Braak staining demonstrates the accumulation of phosphorylated tau, such as neurofibrillary tangles and glial inclusions. Gallyas silver staining (Fig. 3H) in aged 3xTg AD mice $(n=6)$ between 19- and 22months-of-age reveals NFTs throughout the entire brain parenchyma, with strongly stained inclusions in $\mathrm{dSub}$ and EC. However, since tau deposition does not begin in the superficial layers of LEC in this mouse model as it does in patients, a group of mice was injected with MAPT $_{P 301 L}$ human tau (huTau) into this region to better mimic spatiotemporal spread of tauopathy. 
bioRxiv preprint doi: https://doi.org/10.1101/2022.01.14.476357; this version posted January 14, 2022. The copyright holder for this preprint

(which was not certified by peer review) is the author/funder. All rights reserved. No reuse allowed without permission.

A)

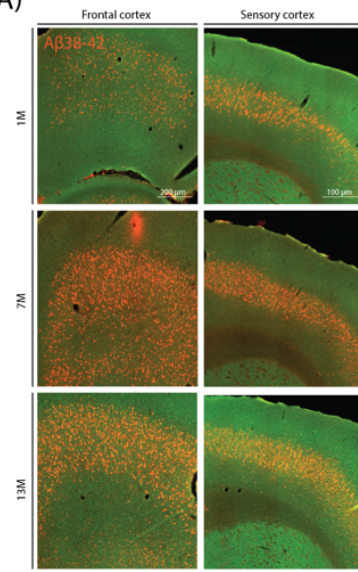

C)
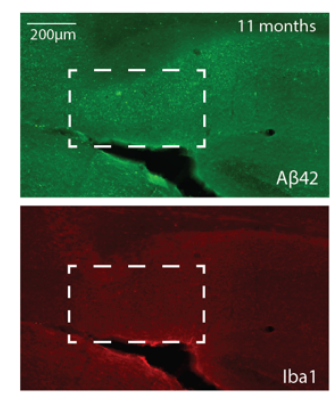

E)
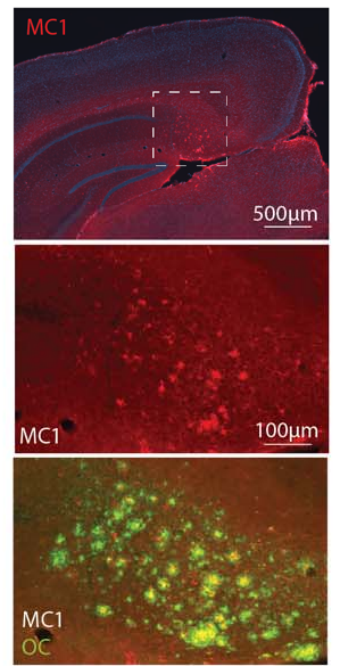

B)
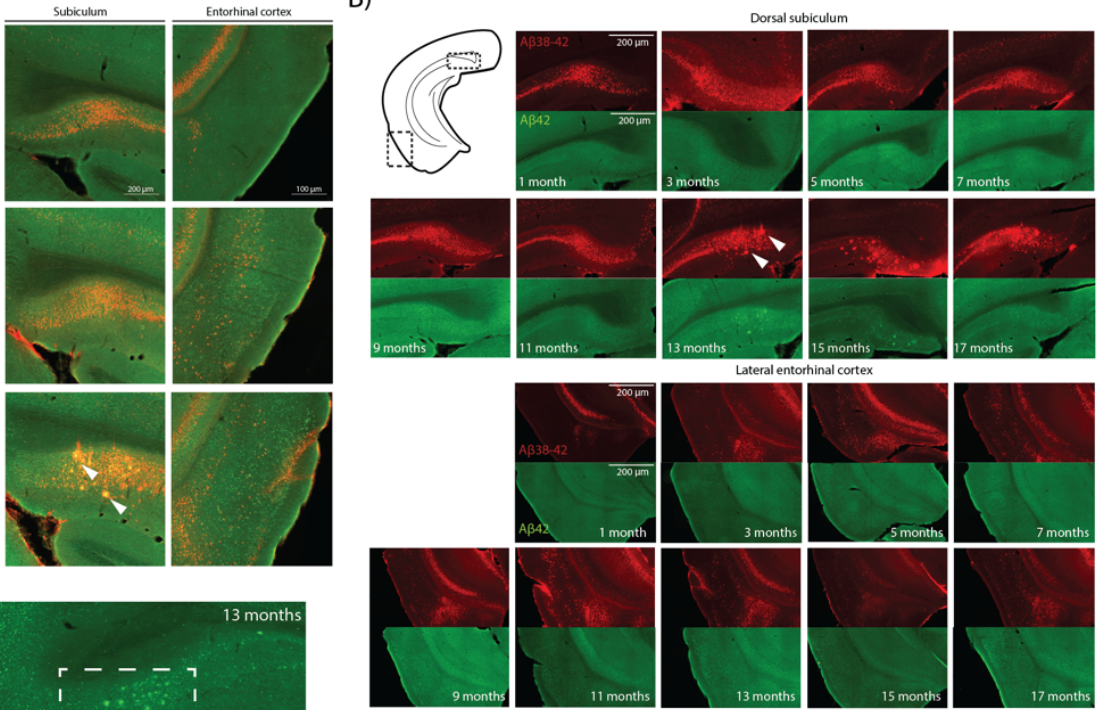

D)
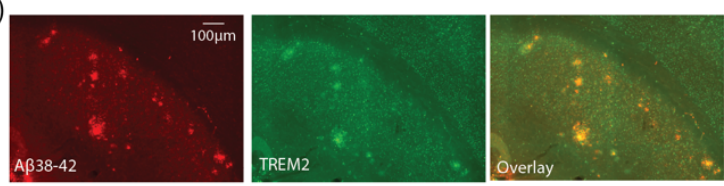

G)
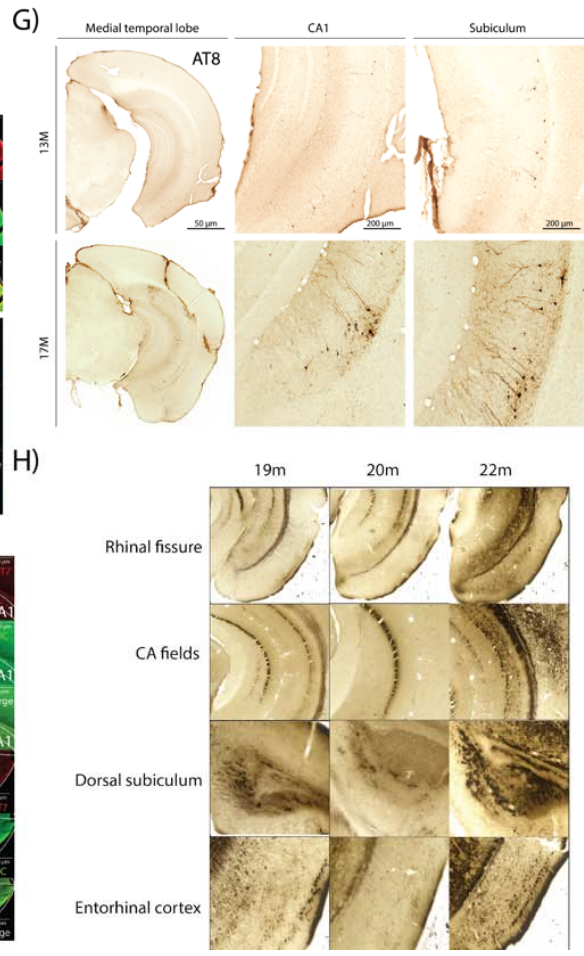

Figure 3. The 3xTg AD mouse model displays Alzheimer's disease-related neuropathology. A) A $338-42$ immunoreactivity in frontal cortex, sensory cortex, Sub, and EC in the 3xTg AD mouse model at 1, 7 and 13-months-of-age. A $\beta 38-42$ (McSA1; red) and $A \beta 42$ (IBL $A \beta 42$; green) immunoreactivity in the 3xTg AD mouse model. Amyloid plaques immunoreactive to A $\beta 38$ 42 (McSA1 antibody) are first apparent at 13 months of age in subiculum. B) A $338-42$ immunoreactivity in the $3 x T g$ AD mouse model at various ages in the dSub and LEC. A $\beta 38-40$ (McSA1; red) and A $\beta 42$ (IBL A $\beta 42$; green) immunoreactivity in the 3xTg $\mathrm{AD}$ mouse model. According to the $\mathrm{ABC}$ scoring system, scoring of diffuse amyloid plaques is assessed in the cerebral cortex, hippocampus, striatum, midbrain, brainstem, and cerebellum according to protocols established by Thal (33) resulting in a Thal phase 0-5, which is translated into the NIA-AA score of A0-A3. Amyloid plaques immunoreactive to A $338-42$ (McSA1 antibody) are first apparent at 13 months of age in dSub, whereas plaques immunoreactive to A $\beta 42$ (IBL A $\beta 42$ antibody) are first 
apparent at 15 months of age in dSub. C) A $\beta 42$ (IBL A 442 ; green) and Iba1 (microglial marker; red) immunoreactivity in dSub at 11 and 13-months-of-age in the 3xTg AD mouse model. D) Aß38-42 (McSA1; red) and TREM2 (microglial receptor; green) immunoreactivity in dSub at 13-months-of-age. E) Conformation-specific tau (MC1; red) and fibrillar A (OC; green) immunoreactivity in dSub at 13-months-of-age. F) Endogenous MAPT (HT7; red) and fibrillar A $(\mathrm{OC}$; green) immunoreactivity in the entire brain at 1-month-of-age in the 3xTg AD mouse model. G) AT8 (detects phosphorylated tau proteins at serine 202 and threonine 205 residues; $\mathrm{DAB}$ ) immunoreactivity in the $3 \times \mathrm{TT}$ AD mouse at 13 and 17-months-of-age. An $\mathrm{ABC}$ score for NFTs is determined in the trans-entorhinal area, corpora ammonis, fronto-parietal cortex, and primary visual cortex to generate a Braak stage (34), which is translated into the NIA-AA score of B0-B3. Most existing mouse models do not generate NFTs, so the National Institutes of Health (NIH) have developed a modified B score for p-tau pathology, including distribution of cytoplasmic neuronal tau such as pre-tangles and threads. H) Gallyas silver staining in the 3xTg AD mouse at 19, 20 and 22-months-of age. Abbreviations; M: month(s); A $\beta$ : amyloid- $\beta$; Sub: subiculum; dSub: dorsal subiculum; LEC: lateral entorhinal cortex; S1: primary somatosensory cortex; S2: secondary somatosensory cortex; Olf: olfactory area; OC: orbital cortex; PrL: prelimbic cortex; Cing: cingulate cortex; M1: primary motor cortex; M2: secondary motor cortex; FC3: frontal cortexarea 3; Ins: insular cortex; CA1: cornu ammonis field 1; CA2: cornu ammonis field 2; CA3: cornu ammonis field 3; Amy: amygdala; diEC: dorsal intermediate entorhinal cortex; dIEC: dorsolateral entorhinal cortex; dSub: dorsal subiculum; PER: perirhinal cortex.

\section{Fasudil and Lonafarnib infusions reduced amyloid- $\beta$ and tau}

As Lonafarnib has previously been shown to increase the activation of lysosomes (26), we hypothesized that Lonafarnib infusions would increase lysosomal associated membrane protein 1 (LAMP1) immunoreactivity after infusions in 3xTg AD mice. LAMP1 immunoreactivity was more prominent in the dSub of Lonafarnib infused mice $(n=2)$ compared to saline infused mice $(n=2)$, particularly in amyloid plaques in the dSub (Fig. 4A). Fasudil infusions reduced the number of intracellular A $\beta$-positive neurons in dSub in young 3xTg mice (Fig. 4B; $n=3 ; t_{18}=$ 2.634, $P=0.0169$, unpaired two-tailed t-test). Lonafarnib did not affect the number of intracellular A $\beta$-positive neurons in dSub in young 3xTg AD mice (Supplementary Fig. 2). However, Lonafarnib infusions appear to have slightly reduced the number of human tau (huTau) neurons in lateral EC after injection of P301L mutated tau (Fig. 4C; $n=4 ; n$.s.). In older $3 \times \mathrm{xTg}$ AD mice, Fasudil infusions reduced the number (n.s.) and size $\left(t_{26}=4.685, P<0.0001\right.$, unpaired two-tailed t-test) of amyloid plaques, whereas Lonafarnib infusions significantly reduced the number $\left(t_{14}=3.861, P=0.0017\right.$, unpaired two-tailed t-test), but not size (n.s.) of amyloid plaques in $3 \times \mathrm{xTg}$ AD mice (Fig. 4D; $n=5$ ). In older animals, Lonafarnib infusions increased A $\beta 40$ and t-tau levels in CSF (Supplementary Fig. 3). Lonafarnib infusions appear to lower MCI-positive neurons in dSub after amyloid plaque deposition in 3xTg AD mice (Fig. 4E; $n=2$ ). Moreover, Fasudil infusions decreased the number and size of dense-core amyloid plaques, whereas Lonafarnib decreased the number but increases the size of these forms of amyloid plaques (Supplementary Fig. 4). Fasudil and lonafarnib infusions both decreased A $\beta 40$ and t-tau levels in CSF (Fig. 4F, G). 
bioRxiv preprint doi: https://doi.org/10.1101/2022.01.14.476357; this version posted January 14, 2022. The copyright holder for this preprint (which was not certified by peer review) is the author/funder. All rights reserved. No reuse allowed without permission.

A)
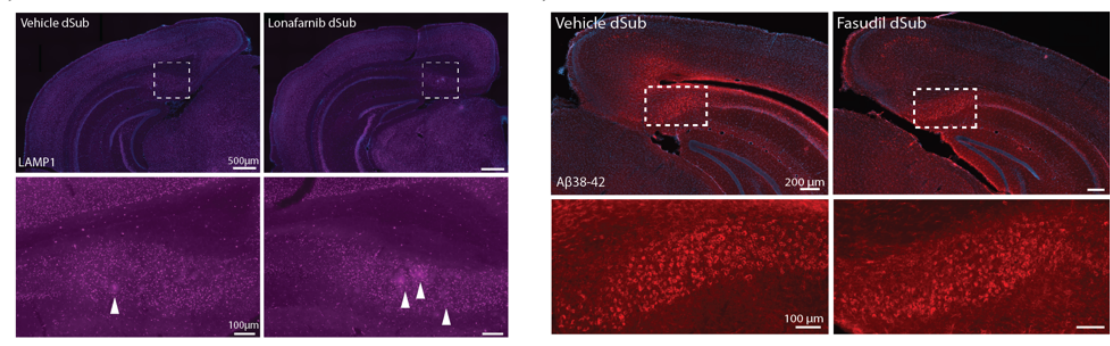

C)
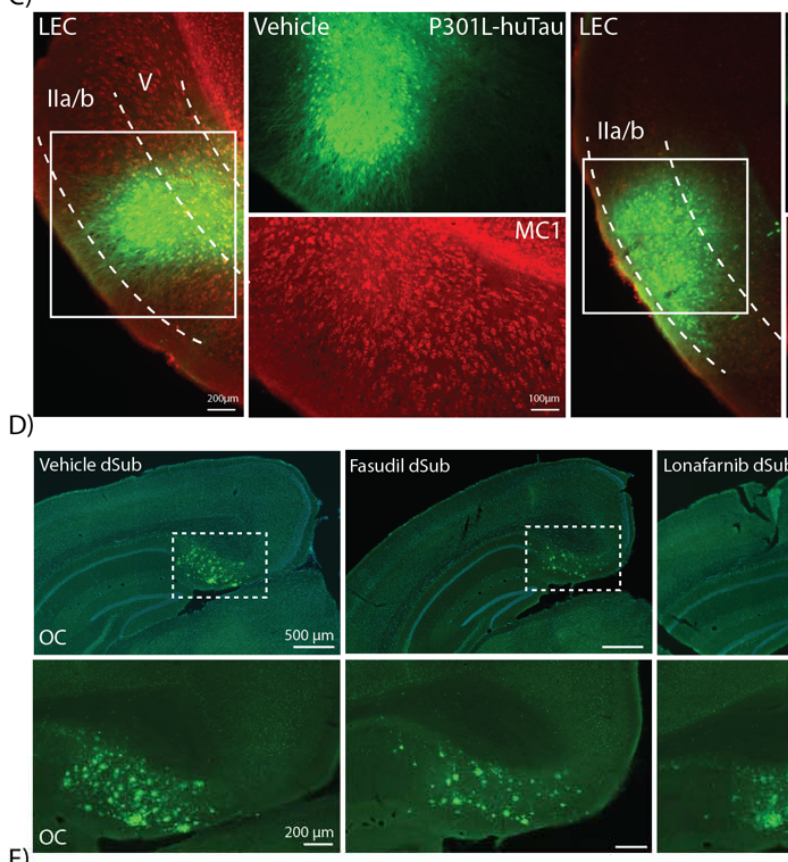

E)

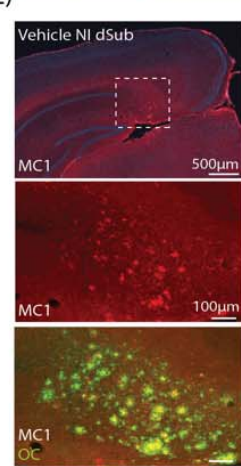

-
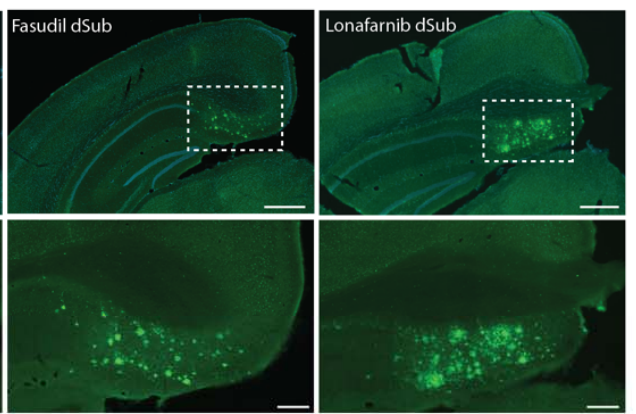

Figure 4. Fasudil and Lonafarnib infusions reduced Aß and tau. A) LAMP1 (lysosomal marker; purple) immunoreactivity (arrowheads) in dSub in $3 \mathrm{xTg} \mathrm{AD}$ mice $(n=4)$ receiving infusions of a vehicle or Lonafarnib. B) A $\beta 38-42$ (McSA1; red) immunoreactivity in $\mathrm{dSub}$ in $3 \mathrm{xTg} \mathrm{AD}$ mice $(n=3)$ receiving infusions of a vehicle or Fasudil. Mean number of intracellular $\mathrm{A} \beta+$ neurons and SD displayed in bar graph, unpaired two-tailed t-test. C) P301L tau injected (GFP; green) 3xTg mice AD ( $n=$ 4) receiving infusions of a vehicle or Lonafarnib and MC1 (conformation-specific tau; red) immunoreactivity in lateral EC. Mean number of huTau+ neurons and SD displayed in bar graph, unpaired two-tailed t-test. D) Amyloid plaques (OC; green) in dSub of $3 \mathrm{xTg} \mathrm{AD}$ mice $(n=5)$ receiving infusions of a vehicle, Fasudil or Lonafarnib. Mean number and size of amyloid plaques and SD displayed in bar graph, unpaired two-tailed t-test. E) MC1 (conformation-specific tau; red) and OC (amyloid plaques; green) immunoreactivity in 3xTg AD mice $(n=2)$ receiving infusions of a vehicle or Lonafarnib in infused (I) and non-infused (NI) hemispheres. F) Treatment with sterile saline or Fasudil on day 8 in 7-month-old 3xTg AD mice $(n=4)$. Bar graph displays mean $\mathrm{pg} / \mathrm{ml}$ levels of A $\beta 40$ and t-tau in CSF, error bars = SD. G) Treatment with sterile saline or Lonafarnib on day 9 in 6-month-old 3xTg AD mice $(n=4)$. Bar graph displays mean $\mathrm{pg} / \mathrm{ml}$ levels of A $\beta 40$ and t-tau in CSF, error bars = SD. Abbreviations; dSub: 
dorsal subiculum; $A \beta$ : amyloid- $\beta$ : iA $\beta$ : intracellular amyloid- $\beta$; LEC: lateral entorhinal cortex; huTau: human tau; NI: non-

234 infused; I: infused.

\section{Co-infusion of Fasudil and Lonafarnib is more effective in reducing neuropathology}

237 Since both drugs appeared to either reduced A $\beta$ (Fasudil) or tau (Lonafarnib) during our pilot 238 experiments, we did co-infusions of both drugs and assessed intracellular $A \beta$ accumulation in the $\mathrm{dSub}$ of 3xTg AD mice. Co-infusions of both drugs greatly reduced the number of intracellular A $\beta$-positive neurons in dSub (Fig. 5A; $n=11 ; U=3.54 .5, P<0.0001$, Mann-Whitney U test). Infusions by in vivo microdialysis were more effective in reducing intracellular $A \beta$ accumulation in the dSub compared to oral administration of the drugs (Fig. 5B; $n=3 ; t_{65}=2.537, P=0.0136$, unpaired two-tailed t-test). Co-infusion of both drugs reduced A $\beta 40$ and t-tau levels in CSF (Fig. 5C). Co-infusion of both drugs appeared to slightly improve memory when tested in a contextual memory paradigm (Fig. 5D; $n=3$ ). Animals infused with a vehicle and those infused with drugs were able to complete morphed contextual experimental testing and were therefore tested in the Squircle context (35). Mice infused with drugs were slightly better at digging in the reward

248 locations associated with training in a square and a circle compared to animals infused with a 249 vehicle. 
A)
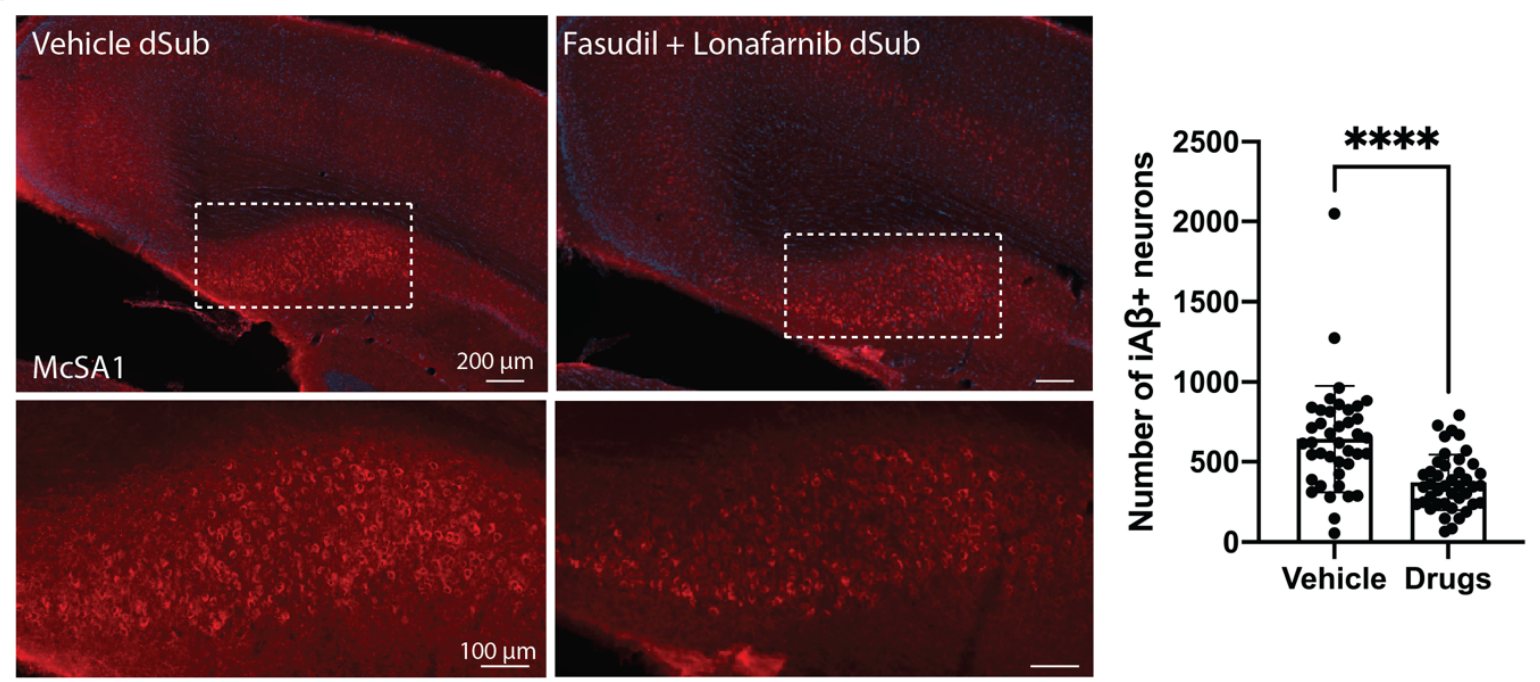

B)

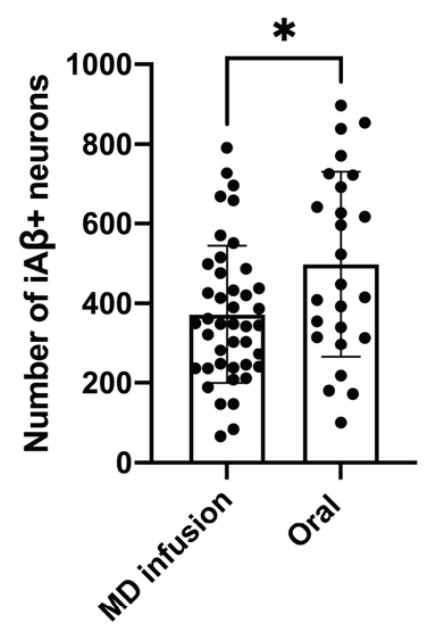

C)

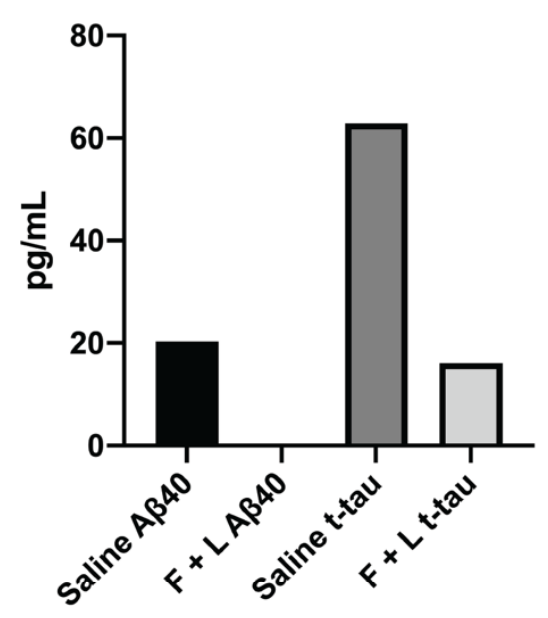

D)

Average across all days

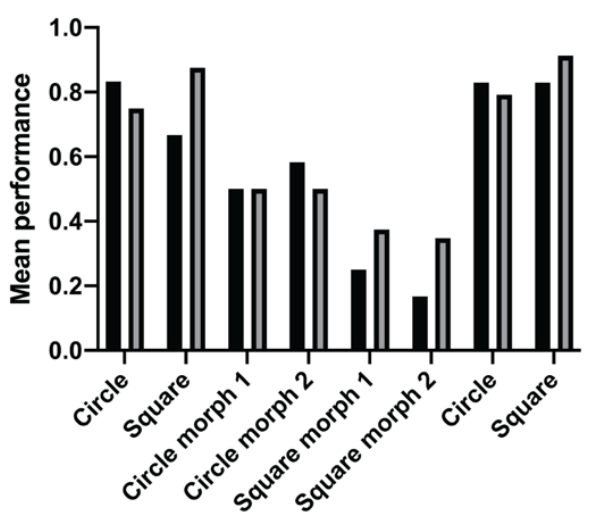

Squircle task
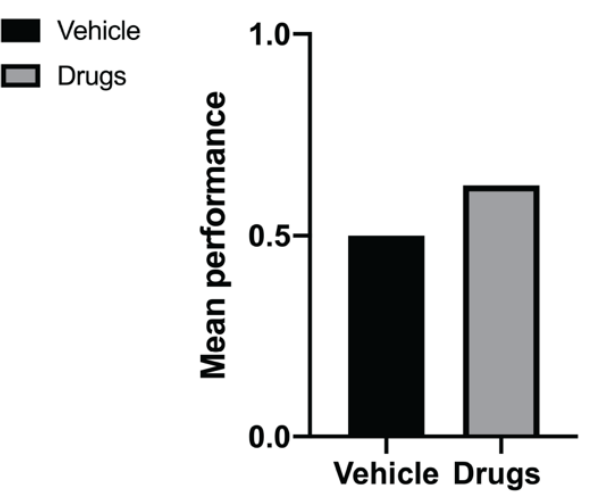

Figure 5. Co-infusion of Fasudil and Lonafarnib is more effective in reducing neuropathology. A) A $338-42$ (McSA1; red) immunoreactivity in $\mathrm{dSub}$ in $3 \times \mathrm{Tg} \mathrm{AD}$ mice $(n=11)$ receiving infusions of a vehicle, or Lonafarnib and Fasudil. Median number of intracellular A $\beta+$ neurons and IQR displayed in bar graph, Mann-Whitney $U$ test. B) Mean number of intracellular A $\beta+$ neurons in dSub after infusions of Lonafarnib and Fasudil via in vivo microdialysis $(n=11)$ or oral administration $(n=3)$. Mean number of intracellular $A \beta+$ neurons and SD displayed in bar graph, unpaired two-tailed t-test. C) Treatment with sterile saline or 
Fasudil + Lonafarnib on day 7 in 1-month-old 3xTg AD mice $(n=4)$. Bar graph displays mean pg/ml levels of A $\beta 40$ and t-tau in CSF. D) Mean performance in morphed contexts across all days and performance in the Squircle context in $3 \times \mathrm{Tg}$ mice $\mathrm{AD}(n=3)$ receiving either a vehicle or co-infusion of Fasudil and Lonafarnib. Abbreviations; dSub: dorsal subiculum; iA $\beta$ : intracellular amyloid- $\beta$; MD: microdialysis.

\section{DISCUSSION}

Before proceeding with drug administration, it was important to establish how well the specific mouse model recapitulates key hallmarks of human AD pathology. Our findings support that the $3 x T g$ AD mouse model captures AD-related neuropathology, with intracellular $\mathrm{A} \beta$ appearing first in the HPC, where subsequent amyloid plaques are deposited at later stages. According to previous characterizations, intracellular $A \beta$ immunoreactivity should be present in CA1, amygdala and neocortex at 4 months of age $(22,36)$, whereas here we found it to be observed in frontal and sensory areas, the HPC, and parts of the cerebellum as early as 1 month of age. Earlier characterizations of this mouse line have observed amyloid plaque deposits in the frontal cortex at 6 months of age $(22,36)$. The present findings are contradictory to earlier characterization, as amyloid plaques are observed first at 13 months of age in the dSub. Amyloid plaques deposited in dSub of the 3xTg AD mouse model are associated with TREM2-related immunoreactivity. Moreover, $\mathrm{MC} 1$ immunoreactivity within OC-positive amyloid plaques gives support to the interplay between $A \beta$ and tau in $A D$ pathophysiology (37). Previous findings have shown that aggregates of hyperphosphorylated tau in this mouse line is present in CA1 at 12-15 months of age (22), whereas here we found it to be present in frontal and sensory areas, and the HPC as early as 1 month of age. This mouse model harbors endogenous tau that leads to subsequent NFT development in subiculum in older ages. There are NFTs present in pyramidal CA1 neurons at 18 months of age, however there are fewer NFTs than reported by Oddo and colleagues (22). Overall, this mouse model presents as a valid model for studying the synergistic effects of $A \beta$ and tau in $A D$.

300 Early postmortem studies of brains from $\mathrm{AD}$ patients suggested that initial accumulation of $\mathrm{A} \beta$

Treatment with Fasudil reduced intracellular A $\beta$ in the brain and CSF A $\beta$, as well as the number and size of amyloid plaques in HPC. This is in line with the notion that when the Wnt signaling pathway is inhibited it reduces $A \beta$ production (38-40). Treatment with this drug also reduced ttau levels in CSF. Lonafarnib, on the other hand, reduced conformation-specific tau in LEC following injections of P301L huTau and the number of amyloid plaques in HPC. This lends support to findings that suggest that the expression of autophagic proteins decline with age, which may impact neurodegeneration in $\operatorname{AD}(41,42)$. Treatment with this drug also reduced $\mathrm{A} \beta$ and t-tau levels in CSF. On the other hand, we can only speculate that the finding of larger amyloid plaques after treatment with Lonafarnib could be due to the increase of intracellular digestion, resulting in a larger expansion of amyloid plaques (43). In older animals, treatment with Lonafarnib increased A $\beta$ levels in CSF. Both drugs targeted dense-core amyloid plaques, and these depositions are associated with microglial activation, subsequent neurodegeneration, and cognitive decline in human patients $(44,45)$. Intriguingly, we found that co-infusion of both drugs greatly reduced intracellular A $\beta$ in HPC and positively affected contextual memory performance in mice.

occurs in the neocortex with subsequent spread of aggregates to deeper structures such as those in the medial temporal lobe (33). However, during postmortem studies, reports are unable to 
document $\mathrm{A} \beta$ deposition over time prior to the development of $\mathrm{AD}$-related dementia. Recent advances in positron emission tomography (PET) imaging have enabled longitudinal human $A \beta$ imaging studies that confirm the importance of cortical $A \beta$ in the diagnosis and prediction of progression of cognitive impairment $(46,47)$, although they have largely not examined deeper structures for $\mathrm{A} \beta$ load and spread $(47,48)$. Therefore, a systematic and comprehensive approach to studying $A \beta$ deposition may reveal unexplored $A \beta$-related changes in the brain of $A D$ patients (49). In line with this, recent findings suggest that subcortical memory network hubs may be critically susceptible to pathological changes that occur in $\mathrm{AD}$ and that changes within these networks may contribute to cognitive decline (50). Regarding A $\beta$ spread, the dSub, and other hippocampal regions, are involved prior to the EC in the $3 \times \mathrm{Tg}$ AD mouse model. Our characterization results also suggest that early intracellular $A \beta$ might be in monomeric or fibrillar forms, and not oligomeric, and the latter forms are better correlated with disease severity than are amyloid plaques containing insoluble $\mathrm{A} \beta$ fibrillary species $(51,52)$. These latter results are in line with the notion that $A \beta$ accumulates over decades in human patients without affecting cognitive decline prior to the deposition of amyloid plaques (53).

Several factors suggest that simultaneous treatment with Fasudil and Lonafarnib would be more beneficial than either drug alone. For instance, amyloidogenic processing of APP occurs in endosomes, following clathrin-mediated endocytosis (54), and endosomal trafficking of familial $\mathrm{AD}$ forms of $A P P$ has been shown to differ from that of wild-type APP (55). Interestingly, WntPCP signaling is also tightly linked to clathrin-mediated endocytosis (56). This follows our findings of reduced dense-core amyloid plaques in mice after Fasudil treatment. Moreover, researchers have found that clathrin-positive endosomes containing $A \beta$ are involved in a process of LC3-associated endocytosis (57). This endocytic pathway was found to be a critical regulator of microglia for removal of protein aggregates in mice modelling AD. Mice deficient in LC3associated endocytosis displayed accelerated neurodegeneration, impaired neuronal signalling, and memory deficits. This seems to be in line with our characterization of the $3 \times \mathrm{Tg}$ AD mouse model and our observation of TREM2-positive microglia clustering around amyloid plaques.

330 Push-pull microdialysis offers a powerful method for the simultaneous collection of CSF and infusion of disease-targeting drugs, however, there are potential limitations that should be considered. Recovery rates, which are inversely proportional to the perfusate flow rate $(58,59)$, for all four CSF biomarkers (A $\beta 40, A \beta 42$, t-tau, p-tau) were relatively low compared to previous findings (60) for longitudinal CSF biomarkers in the 3xTg AD mouse model, which was likely due to a relatively fast sampling rate $(1 \mu \mathrm{L} /$ minute). We have previously found that a sampling rate of $0.2 \mu \mathrm{L} /$ minute was ideal for the recovery of CSF $\mathrm{A} \beta$ and tau (60). However, due to the repeated infusions of drugs in animals in the current study, a faster sampling rate was used to reduce infusion time per animal as well as to administer the drugs as one dose, rather than as a slow diffusion process over many hours. Additionally, as CSF t-tau levels generally increased in the first few days of sampling, it is likely that the removal and insertion of the microdialysis dummy and sampling probe resulted in minor tissue damage at the start of sampling. This is consistent with the fact that in response to acute brain injury, CSF t-tau concentrations increase in the first few days following injury, then reduce over time (61). However, we have previously shown that long-term implantation of our microdialysis probe does not lead to increased neuroinflammation in 3xTg AD mice (60).

346 Moreover, intracerebral microdialysis is an invasive technique, so it is currently only used in patients requiring neurocritical care, neurosurgery, or brain biopsy (62). A leaky BBB often 
precedes amyloid plaque formation in $\mathrm{AD}$ patients (63), and this is also observed in $3 \times \mathrm{Tg} \mathrm{AD}$ mice (64). Enlarged ventricles and concomitant brain atrophy could be a possible indicator of $\mathrm{AD}$ disease progression (65). In line with this, a study has shown that in the $3 \times \mathrm{Tg}$ AD mouse model, ventricles become enlarged compared to control mice already at 2 months-of-age (66). Moreover, in our study, and most preclinical translational AD research, there is an increased focus on familial rather than sporadic forms of $\mathrm{AD}$. Whereas most $\mathrm{AD}$ cases are sporadic, transgenic animal models of $\mathrm{AD}$ are based on genetic mutations found in familial $\mathrm{AD}$, specifically in the APP, presenilin 1 (PSEN1) and presenilin 2 (PSEN2) genes (67). This limitation, however, should not detract from the value of the current study and other studies using transgenic models, as the continuum of sporadic and familial forms of AD are very similar, except for age-of-onset (67).

\section{Conclusions and future directions}

Due to the current failure in disease-targeting drugs to attenuate AD neuropathology, there is an urgent need for novel therapeutic approaches. Here we repurposed two FDA-approved drugs, Fasudil and Lonafarnib, with independent actions on biochemical events leading to AD pathology. Treating 3xTg AD mice with both drugs simultaneously was more effective at reducing intracellular $A \beta$ and led to better contextual memory performance. However, an unexpected finding was that Lonafarnib treatment increased amyloid plaques and CSF A $\beta$ in old animals, suggesting that activating the endosomal-lysosomal system may inadvertently increase $\mathrm{A} \beta$ pathology if administered too late in the $\mathrm{AD}$ continuum. In line with this, it may be interesting to infuse Lonafarnib prior to over-expression of huTau in lateral EC. Future studies should aim to longitudinally infuse both drugs and examine the effect on AD pathophysiology. Furthermore, there is currently an urgent need within the field to develop blood-based biomarkers which are inexpensive and minimally invasive, which can detect early neuropathological changes in $\mathrm{AD}(68)$. It is important to note, however, that blood biomarkers have lower sensitivity and specificity than CSF biomarkers, and this can be attributed to the fact that the BBB prevents diffusion of analytes into the blood via a filtering mechanism (69), and there is currently no approved blood biomarker for AD. Taken together, our findings lend support to the application of repurposed drugs to attenuate Alzheimer's disease neuropathology at various therapeutic time windows in preclinical models and thus obtain insights into

\section{MATERIALS AND METHODS}

\section{Animals}

383 Thirty 3xTg AD mice and two control B6129 mice were included in these experiments. These 384 mice contain three mutations associated with familial Alzheimer's disease $\left(A P P_{S w e}, M A P T_{P 301 L}\right.$, and $\left.P S E N 1_{M 146 V}\right)$. The $3 \times T$ TD mouse model is based on a C57BL/6;129X1/SvJ;129S1/Sv (Jackson Laboratory, Bar Harbor, ME, USA) background. The model exhibits both amyloid plaques and NFTs, and cognitive impairment appear at 4 months of age in these mice.

The donating investigators of the 3xTg AD mouse model communicated to Jackson Laboratories that male transgenic mice may not exhibit the phenotypic traits as originally described (36). Therefore, only female mice were included in these experiments. All housing and breeding of 
animals used was approved by the Norwegian Animal Research Authority and is in accordance with the Norwegian Animal Welfare Act $\S \S 1-28$, the Norwegian Regulations of Animal Research $\S \S 1-26$, and the European Convention for the Protection of Vertebrate Animals used for Experimental and Other Scientific Purposes (FOTS ID 21061). The animals were kept on a $12 \mathrm{~h}$ light/dark cycle under standard laboratory conditions $\left(19-22^{\circ} \mathrm{C}, 50-60 \%\right.$ humidity), and had free access to food and water.

\section{Microdialysis guide implantation surgeries}

Surgeries were based on our earlier established protocol $(60,70)$, but in brief, implantation surgery was performed to insert microdialysis guide cannulas (CMA 7; CMA Microdialysis AB, Kista, Sweden) into the lateral ventricle of mice. Mice were anesthetized with isoflurane gas (4 $\%$ induction and 1.5-3 \% maintenance; IsoFlo vet., Abbott Laboratories, Chicago, IL, USA) prior to being fixed in a stereotaxic frame (Kopf Instruments; Chicago, IL, USA). Prior to making any incisions, Marcain (0.03-0.18 mg/kg; Aspen Pharma, Ballerup, Denmark) was injected subcutaneously into the scalp and Metacam $(5 \mathrm{mg} / \mathrm{kg}$; Boehringer Ingelheim Vetmedia, Copenhagen, Denmark) and Temgesic (0.05-0.1 mg/kg; Invidor UK, Slough, Great Britain) were administered subcutaneously for intraoperative pain relief. Equal heights of bregma and lambda were measured to ensure that the skull was level for each animal (with $\pm 0.1 \mathrm{~mm}$ tolerance), as well as 2 points equally distant from the midline. After levelling the skull, the stereotaxic coordinates were derived to target the lateral ventricle $(\mathrm{A} / \mathrm{P}-0.1 \mathrm{~mm}, \mathrm{M} / \mathrm{L}+1.2 \mathrm{~mm}, \mathrm{D} / \mathrm{V}-2.75$ $\mathrm{mm})$. The microdialysis guide cannula was attached to the stereotaxic frame using a guide clip and connection rod for the clip (CMA Microdialysis AB, Kista, Sweden). The skull was drilled through at these coordinates and the guide cannula was slowly lowered into the drilled hole. The guide cannula was attached to the skull with super glue and dental cement (Dentalon Plus; Cliniclands AB, Trelleborg, Sweden). Post-surgery, Metacam and Temgesic were administered within 24 hours. The guide cannula was implanted into the right hemisphere of all animals, as we did not observe any lateralization of pathology in the brains of 3xTg AD mice (Supplementary Fig. 5).

\section{Stereotaxic viral injections of P301L tau}

Mice were treated identically to microdialysis guide implantation surgeries, up until deriving stereotaxic coordinates. A craniotomy was made at $0.5 \mathrm{~mm}$ anterior to lambda and $\sim 4 \mathrm{~mm}$ lateral (dependent on animal weight) to the midline. A Hamilton microsyringe (Neuros 32-gauge syringe, $5 \mu$, Hamilton company, Nevada, USA) was lowered vertically into the brain to a depth $\sim 3.6 \mathrm{~mm}$ (dependent on animal weight) from the surface, and 2000-2500 $\mathrm{nL}$ of viruses was injected using a microinjector (Nanoliter 2010, World Precision Instruments Inc., USA). We used AAV8-P301L Tau-2a-GFP for injections into layer II of lateral EC (generated in house by Dr. Nair). The microsyringe was kept in place for 5 minutes prior and after the injection, to minimize potential upward leakage of the viral solution. Metacam was given within 24 hours post-surgery. Animals were implanted with microdialysis guide cannulas two months following 432 injections.

\section{Push-pull microdialysis apparatus and sampling}


Push-pull microdialysis was conducted as previously described $(60,71)$, but in brief a refrigerated fraction collector (CMA 470) was set to $6{ }^{\circ} \mathrm{C}$ for the storage of collected CSF in 300 $\mu 1$ low-retention polypropylene plastic vials (Harvard Apparatus, Cambridge, MA, USA). Fluorinated ethylene propylene (FEP) peristaltic tubing (CMA Microdialysis AB, Kista, Sweden) was placed inside each plastic vial for collection and connected to the cassette of the peristaltic roller pump (Reglo ICC Digital). This peristaltic FEP tubing was connected to the outlet side of microdialysis probes ( $\beta$-irrigated $2 \mathrm{mDa}$ microdialysis probe; CMA 7; CMA Microdialysis AB, Kista, Sweden) with a polyethersulfone $2 \mathrm{~mm}$ membrane with tubing adapters bathed in $75 \%$ ethanol. FEP tubing (CMA Microdialysis AB, Kista, Sweden) was connected to each microsyringe. The FEP tubing was then connected to the inlet part of the microdialysis probes. Transparent cages were prepared with $1.5 \mathrm{~cm}$ of bedding, filled water bottles, and treats. Saline or drugs loaded inside a gastight microsyringe (CMA Microdialysis AB, Kista, Sweden), which was placed into a syringe pump (CMA 4004). The 'dead volume' of the FEP outlet tubing (1.2 $\mathrm{mL} / 100 \mathrm{~mm}$ ) was calculated. $100 \mathrm{~cm}$ of FEP outlet tubing was used, and therefore the first $12 \mathrm{~mL}$ sampled from each animal were discarded. Prior to inserting the microdialysis probes into the guide cannula, the probe was conditioned in $75 \%$ ethanol for better recovery of analytes. At the conclusion of microdialyte sampling, the vials of $60 \mu \mathrm{l} \mathrm{CSF}$ were centrifuged and kept at $-80{ }^{\circ} \mathrm{C}$ until the samples were analyzed with multiplex ELISA.

\section{Drug infusions into the lateral ventricle}

454 Fasudil is a promising drug candidate for AD as it has previously been given clinical approval for the treatment of cerebral vasospasm and is therefore safe to use in humans. Previously, researchers have administered Fasudil at $10 \mathrm{mg} / \mathrm{kg}$ daily using DMSO as a vehicle into the lateral ventricle for 14 days (24). However, DMSO can damage the BBB, mitochondria and can cause apoptosis (72) and therefore all drugs used in experiments were diluted in sterile saline for delivery. Because we had a less effective delivery vehicle, Fasudil (Selleck Chemicals, Houston, TX, USA) in powder form $(10 \mathrm{mM})$ was diluted in sterile saline for a final concentration of $50 \mathrm{mg} / \mathrm{kg}$ and stored at $-80 \square$ between infusion experiments.

Previous research has administered Lonafarnib, which is also approved for cancer treatment in humans, orally at $80 \mathrm{mg} / \mathrm{kg}$ for five days on and five days off for a total of 10 weeks (26). For the drug to diffuse into the brain parenchyma, the same dosage was infused into the lateral ventricle in the current experiments. Lonafarnib (5 mM; Cayman Chemical, Ann Arbor, MI, USA) in powder form was dissolved in sterile saline at $60 \square$ to a final concentration of $80 \mathrm{mg} / \mathrm{kg}$ and stored at $-20 \square$ between infusion experiments.

All dosages in $\mathrm{ml}$ were calculated using; dosage $(\mathrm{mg}) /$ concentration $(\mathrm{mg} / \mathrm{ml})=$ dose $\mathrm{x} \mathrm{ml})$ and were infused at a volume of $60 \mu \mathrm{l}$ at a rate of $1 \mu \mathrm{l} / \mathrm{min}$ using saline as a control vehicle. In one group of animals $(n=3), 0.6 \mathrm{ml}$ of $80 \mathrm{mg} / \mathrm{kg}$ Lonafarnib and $50 \mathrm{mg} / \mathrm{kg}$ Fasudil were mixed into

\section{Contextual memory testing}

474 Starting 5 days before the experiment, animals were taught to dig in a brain cup for a food reward (Nestle chocolate loopie) in their home cage by providing them once daily with the 
contextual paradigm, they were handled, and food deprived for five days prior to training and experimental testing.

The training of the task was conducted in a square $(4 \times 29.25 \mathrm{~cm})$ and circle $(157 \mathrm{~cm}$ circumference) chamber built out of rectangular Legos $(2 \times 1 \mathrm{~cm}$, Lego, Billund, Denmark). The testing of the task was conducted in a hexagon $(6 \times 26.16 \mathrm{~cm})$, octagon $(8 \times 19.63 \mathrm{~cm})$, and Squircle $(2 \times 78.5 \mathrm{~cm})$ chambers built out of rectangular Legos $(2 \times 1 \mathrm{~cm})$. All chambers were 15 $\mathrm{cm}$ tall. The same base was used for all chambers, and plastic cups were embedded in the base. The plastic cups contained odor-masked bedding, consisting of $1 \mathrm{~g}$ of odor mask (ground ginger) for every $100 \mathrm{~g}$ of bedding. Testing in all chambers occurred in the same location in the experimental room. The chambers were surrounded by a square black curtain with rounded corners, and were uniformly lit from overhead. Four distal cues (a yellow square, a red triangle, a blue circle, and a green diamond all in the same size proportion) were placed on all 4 sides of the curtains. All chambers were built in proportion to each other. All rewards were mirrored in the square and circle chamber, i.e., if the reward location was the top right corner in the square, it would be the top left corner in the circle.

A pilot experiment showed that mice could discriminate the square and circle reward locations to a performance criterion after 16 training trials. Thus, all experiments began with a training phase consisting of four training trials per chamber per day for 2 days, with successive trials alternated across chambers ( 8 trials total in the square chamber and 8 trials total in the circle chamber). During this training, mice were taught to search for a reward (Chocolate Cheerios Cereal, Nestle, Vevey, Switzerland), which was visible for the first two training trials per chamber and buried in the remaining training trials. The correct reward location was assessed by digging, and after correctly digging $66.67 \%$ of the time, animals were ready for testing. If animals dug in the wrong location, the trial ended. If the animal did not dig after 5 minutes, the trial ended. In the square chamber, the reward was always located in the right-hand corner, whereas in the circle chamber, the reward was always located in the left-hand corner. These locations were counterbalanced across animals; however, for all analyses and figures, the percentage of searches at each corner are reflected such that the correct corner is the same for all animals.

All experiments began with a training phase consisting of four training trials per chamber per day for 2 days, with successive trials alternated across chambers $(8$ trials total in the square chamber and 8 trials total in the circle chamber). Animals were disoriented before the start of every trial. To disorient an animal, it was placed inside the hands of the experimenter. The experimenter slowly rotated their hands roughly four full clockwise then four full counterclockwise revolutions. The animal was then carried in the experimenter's hands to the chamber. To ensure that the animal used distal cues, it was placed in the chamber in proportion to each side of the chamber with a $90^{\circ}$ clockwise rotation before each trial, counterbalanced so that all orientations relative to the room were experienced equally often. The chambers were cleaned with ethanol at the end of each trial to remove odor trails. The intertrial interval was 5 $\min$.

516 Following training, animals were tested in eight sessions per day for 4 days. The first two trials consisted of animals being tested in the square and circle chamber with rewards. In trials 3 to 6 , the animals were tested in two morphed chambers (a morphed square and circle chamber). The order of the morphed chambers was randomized across days and balanced across animals. In trials 7 and 8, the animals were again tested in the square and circle chambers with rewards in order to not lose motivation. If an animal was able to perform the tasks up until day 4, they were 
tested in the Squircle context on the fifth day. During reward trials, mice were removed from the apparatus after they had found the reward. During unrewarded trials, they were removed after their first dig, or after $5 \mathrm{~min}$ (whichever came later). Digs were counted whenever an animal removed bedding from a cup by using one or both paws.

Dig locations and time spent in these locations were calculated using ANY-maze video tracking system (Stoelting Europe) via an overhead, centrally located camera (DMK 22AUC03 USB 2.0 monochrome industrial camera, The imaging Source Europe, Germany). Paired sample t-tests were used to assess the number of correct digs, and the difference between cohorts. All reported statistics are based on two-tailed significance tests. Animals had access to water ad libitum, but to increase motivation to participate in the task, they were maintained at $90-95 \%$ of their freefeed weight.

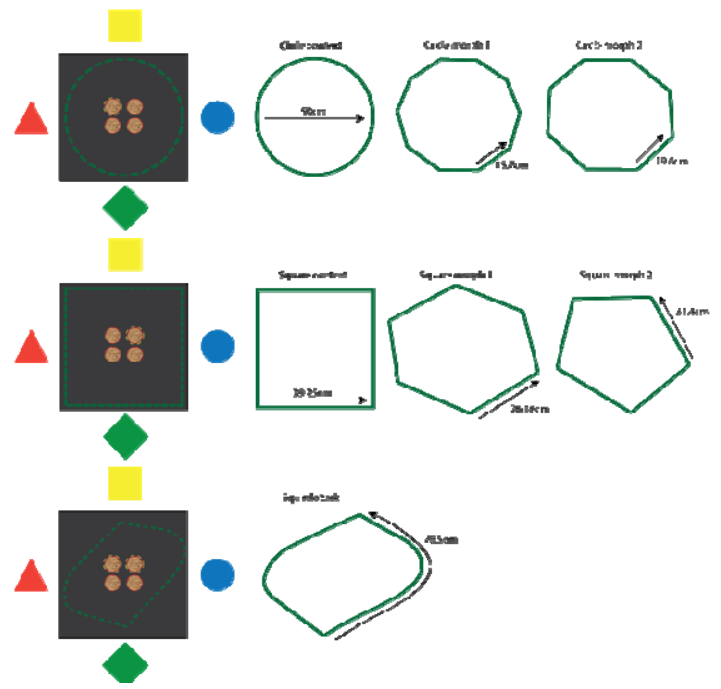

Figure 6. The Squircle task. Mice were initially taught to associate a specific reward location in a square and a circle context. The reward was embedded in one of four cups with ginger-scented bedding. A trial was assessed as correct if the mice dug in the reward location associated with each chamber. If they passed the training phase (66.6\% correct digging), their contextual memory performance was tested in morph chambers: decagon (circle morph 1), octagon (circle morph 2), hexagon (square morph 1), and a pentagon (square morph 2). Mice were tested in morph chambers for 4 days, with 8 sessions a day. If they were able to complete the morph testing, they were tested in a Squircle chamber on the fifth day. Since the Squircle equally resembles a square and a circle context, a trial was considered correct if the mouse dug either of the reward locations attributable to the square- or circle context. If so, it was attributed as contextual association, if not, it was attributed as visual learning.

\section{Proteomic analysis of amyloid- $\beta$ and tau concentrations in CSF}

The MILLIPLEX® MAP human amyloid beta and tau magnetic bead panel 4-plex ELISA kit (Millipore, Burlington, MA, USA) and the Bio-Plex 200 System instrument (Biorad, Hercules, CA, USA) were used to assess simultaneously the concentrations of $A \beta_{40}, A \beta_{42}$, t-tau, and p-tau (Thr181) in CSF samples. The samples were undiluted and analysed in duplicates. The lower limit of quantification (LLOQ) is shown in Supplementary Fig. 6.

\section{Tissue processing and immunohistochemistry}

Mice were administered a lethal dose of sodium pentobarbital $(100 \mathrm{mg} / \mathrm{ml}$; Apotekforeningen, (PFA, $4 \%$; Sigma $\square$ Aldrich) in $125 \mathrm{mM}$ phosphate buffer (PB). Brains were extracted and fixed 
for a minimum of 24 hours in PFA at $4{ }^{\circ} \mathrm{C}$ and transferred to a $2 \%$ DMSO solution prepared in PB for 24 hours at $4{ }^{\circ} \mathrm{C}$. Brains were sectioned coronally at $40 \mu \mathrm{m}$ on a freezing microtome (Microm HM430, ThermoFisher Scientific, Waltham, MA, USA). An incision was made in the non-implanted hemisphere for visualization of the control hemisphere. Immunohistochemical processing was conducted on tissue, see (73) for detailed protocol, and Supplementary Table 1 for antibodies used.

560 Previous research has indicated that a differential microtubule-associated protein 2 (MAP2) 561 immunolabeling pattern can distinguish dense-core amyloid plaques from diffuse amyloid 562 plaques using DAB (Sigma-Aldrich, St. Louis, MO, USA) as a chromogen (74).

563 One series of each brain were dehydrated in ethanol, cleared in xylene (Merck Chemicals, 564 Darmstadt, Germany) and rehydrated before staining with Cresyl violet (Nissl; 1g/l) for 3 minutes to verify probe placement. The sections were then alternatively dipped in ethanol-acetic acid (5 ml acetic acid in $1170 \%$ ethanol) and rinsed with cold water until the desired differentiation was obtained, then dehydrated, cleared in xylene and coverslipped with entellan containing xylene (Merck Chemicals).

Sections were scanned using a Mirax-midi scanner (objective 20X, NA 0.8; Carl Zeiss Microscopy, Oberkochen, Germany), using either reflected fluorescence (for sections stained with a fluorophore) or transmitted white light (for sections stained with Cresyl Violet; Nissl) as the light source.

\section{Quantification of amyloid- $\beta$ in hippocampus and tau in entorhinal cortex}

Series of sections were chosen randomly and coded to ensure blinding to the investigators. The number of cells containing intracellular $\mathrm{A} \beta$, tau, and amyloid plaques, in dSub and lateral EC of infused and non-infused hemispheres of vehicle-infused 3xTg AD mice $(n=10)$ and druginfused mice $(n=20)$ was estimated with Ilastik (75) using the Density cell counting workflow. dSub and lateral EC was delineated using cytoarchitectonic features in sections with Nissl (Cresyl violet) stain, based on The Paxinos \& Franklin mouse atlas (76). The same surface area and rostrocaudal levels of each brain region was selected, and at least 6 slices were used for each hemisphere. Damaged regions of brain slices were cropped out prior to quantification as this could result in false-positive antibody expression.

\section{Statistics}

594

Effect size (Cohen's D) was calculated based on initial experiments between an experimental group infused with Fasudil and a control group infused with a vehicle and the resulting effects on intracellular A $\beta$-positive neurons in dSub. Based on each group consisting of $n=4$ animals, a large effect size of 1.57 was calculated (77). Most of the dataset we normalized (Shapiro-Wilk test) and therefore two-tailed, unpaired t-tests were used to compare mean differences. For the minor parts of the dataset that were non-normalized, nonparametric statistical tests were used (Mann-Whitney U). Correlations between drug- and vehicle-infused CSF protein levels were tested with the Pearson product moment correlation. All statistical tests and graphs were made in Prism 9 (GraphPad Software Inc., CA, USA). 
Acknowledgments:

597 Thank you to Nora Cecilie Ebbesen for allowing us to reproduce the illustration of the Squircle 598 task.

\section{Funding:}

601 Liaison Committee for Education, Research, and Innovation in Central Norway

602 (Samarbeidsorganet HMN- NTNU) grant 2018/42794

603 Joint Research Committee (Felles Forskningsutvalg - St. Olav's Hospital HF and the Faculty of

604 Medicine, NTNU) grant 2019/26157

606 Author contributions:

607 Conceptualization: CB, IS

608 Methodology: CB, JBJ, AS, IS

609 Investigation: $\mathrm{CB}, \mathrm{MH}$

610 Visualization: $\mathrm{CB}, \mathrm{MH}$

611 Funding acquisition: CB, AS, IS

612 Project administration: CB, AS, IS

613 Supervision: AS, IS

614 Writing - original draft: CB

615 Writing - review and editing: CB, MH, JBJ, AS, IS

616

617 Competing interests:

618 Authors declare that they have no competing interests.

Data and materials availability:

621 All data are available in the main text or the supplementary materials.

\section{References}

$624 \quad$ 1. $\quad$ B. De Strooper, E. Karran, The Cellular Phase of Alzheimer's Disease. Cell 164, 603-615 (2016).

625 2. L. M. Bekris, C.-E. Yu, T. D. Bird, D. W. Tsuang, Genetics of Alzheimer disease. J Geriatr Psychiatry $626 \quad$ Neurol 23, 213-227 (2010).

627 3. J. Hardy, J. Selkoe Dennis, The Amyloid Hypothesis of Alzheimer's Disease: Progress and Problems on the Road to Therapeutics. Science 297, 353-356 (2002).

4. E. J. Mufson, M. D. Ikonomovic, S. E. Counts, S. E. Perez, M. Malek-Ahmadi, S. W. Scheff, S. D. 54-69 (2016). 
5. P.-P. Liu, Y. Xie, X.-Y. Meng, J.-S. Kang, History and progress of hypotheses and clinical trials for Alzheimer's disease. Signal Transduction and Targeted Therapy 4, 29 (2019).

6. L.-K. Huang, S.-P. Chao, C.-J. Hu, Clinical trials of new drugs for Alzheimer disease. Journal of Biomedical Science 27, 18 (2020).

7. B. J. Snider, A. M. Fagan, C. Roe, A. R. Shah, E. A. Grant, C. Xiong, J. C. Morris, D. M. Holtzman, Cerebrospinal fluid biomarkers and rate of cognitive decline in very mild dementia of the Alzheimer type. Arch Neurol 66, 638-645 (2009).

8. P. Buchhave, L. Minthon, H. Zetterberg, A. K. Wallin, K. Blennow, O. Hansson, Cerebrospinal fluid levels of beta-amyloid 1-42, but not of tau, are fully changed already 5 to 10 years before the onset of Alzheimer dementia. Arch Gen Psychiatry 69, 98-106 (2012).

9. C. Bjorkli, A. Sandvig, I. Sandvig, Bridging the Gap Between Fluid Biomarkers for Alzheimer's Disease, Model Systems, and Patients. Front Aging Neurosci 12, (2020).

10. C. R. Jack, D. M. Holtzman, Biomarker Modeling of Alzheimer's Disease. Neuron 80, 1347-1358 (2013).

11. B. Dubois, H. Hampel, H. H. Feldman, P. Scheltens, P. Aisen, S. Andrieu, H. Bakardjian, H. Benali, L. Bertram, K. Blennow, K. Broich, E. Cavedo, S. Crutch, J. F. Dartigues, C. Duyckaerts, S. Epelbaum, G. B. Frisoni, S. Gauthier, R. Genthon, A. A. Gouw, M. O. Habert, D. M. Holtzman, M. Kivipelto, S. Lista, J. L. Molinuevo, S. E. O'Bryant, G. D. Rabinovici, C. Rowe, S. Salloway, L. S. Schneider, R. Sperling, M. Teichmann, M. C. Carrillo, J. Cummings, C. R. Jack, Jr., Preclinical Alzheimer's disease: Definition, natural history, and diagnostic criteria. Alzheimers Dement 12, 292-323 (2016).

12. L. Liu, S. K. Herukka, R. Minkeviciene, T. van Groen, H. Tanila, Longitudinal observation on CSF Abeta42 levels in young to middle-aged amyloid precursor protein/presenilin-1 doubly transgenic mice. Neurobiol Dis 17, 516-523 (2004).

13. A. Lloret, D. Esteve, M.-A. Lloret, A. Cervera-Ferri, B. Lopez, M. Nepomuceno, P. Monllor, When Does Alzheimer's Disease Really Start? The Role of Biomarkers. Int J Mol Sci 20, 5536 (2019).

14. D. J. Selkoe, J. Hardy, The amyloid hypothesis of Alzheimer's disease at 25 years. EMBO molecular medicine 8, 595-608 (2016).

15. L. M. Shaw, H. Vanderstichele, M. Knapik-Czajka, C. M. Clark, P. S. Aisen, R. C. Petersen, K. Blennow, H. Soares, A. Simon, P. Lewczuk, R. Dean, E. Siemers, W. Potter, V. M. Lee, J. Q. Trojanowski, Cerebrospinal fluid biomarker signature in Alzheimer's disease neuroimaging initiative subjects. Ann Neurol 65, 403-413 (2009).

16. Y. P. Pang, P. Quiram, T. Jelacic, F. Hong, S. Brimijoin, Highly potent, selective, and low cost bistetrahydroaminacrine inhibitors of acetylcholinesterase. Steps toward novel drugs for treating Alzheimer's disease. J Biol Chem 271, 23646-23649 (1996).

17. J. Cummings, G. Lee, A. Ritter, M. Sabbagh, K. Zhong, Alzheimer's disease drug development pipeline: 2019. Alzheimer's \& dementia (New York, N. Y.) 5, 272-293 (2019).

18. S. Gauthier, M. Albert, N. Fox, M. Goedert, M. Kivipelto, J. Mestre-Ferrandiz, L. T. Middleton, Why has therapy development for dementia failed in the last two decades? Alzheimers Dement 12, 60-64 (2016).

19. S. Oddo, L. Billings, J. P. Kesslak, D. H. Cribbs, F. M. LaFerla, Abeta immunotherapy leads to clearance of early, but not late, hyperphosphorylated tau aggregates via the proteasome. Neuron 43, 321-332 (2004).

20. Y. Yoshiyama, V. M. Lee, J. Q. Trojanowski, Therapeutic strategies for tau mediated neurodegeneration. $J$ Neurol Neurosurg Psychiatry 84, 784-795 (2013).

21. E. Drummond, T. Wisniewski, Alzheimer's disease: experimental models and reality. Acta Neuropathologica 133, 155-175 (2017).

22. S. Oddo, A. Caccamo, J. D. Shepherd, M. P. Murphy, T. E. Golde, R. Kayed, R. Metherate, M. P. Mattson, Y. Akbari, F. M. LaFerla, Triple-Transgenic Model of Alzheimer's Disease with Plaques and Tangles. Neuron 39, 409-421 (2003).

23. B. L. Tang, Y. C. Liou, Novel modulators of amyloid-beta precursor protein processing. J Neurochem $\mathbf{1 0 0 ,}$ 314-323 (2007). Xia, M. Semenov, P. Morin, N. M. Hooper, R. Porter, J. Preston, R. Al-Shawi, G. Baillie, S. Lovestone, A. Cuadrado, M. Harte, P. Simons, D. P. Srivastava, R. Killick, Amyloid $\beta$ synaptotoxicity is Wnt-PCP dependent and blocked by fasudil. Alzheimers Dement 14, 306-317 (2018). Al-Shawi, C. G. Ballard, P. Simons, R. Killick, A role for APP in Wnt signalling links synapse loss with $\beta$ amyloid production. Translational Psychiatry 8, 179 (2018). 
26. I. Hernandez, G. Luna, J. N. Rauch, S. A. Reis, M. Giroux, C. M. Karch, D. Boctor, Y. E. Sibih, N. J. Storm, A. Diaz, S. Kaushik, C. Zekanowski, A. A. Kang, C. R. Hinman, V. Cerovac, E. Guzman, H. Zhou, S. J. Haggarty, A. M. Goate, S. K. Fisher, A. M. Cuervo, K. S. Kosik, A farnesyltransferase inhibitor activates lysosomes and reduces tau pathology in mice with tauopathy. Science Translational Medicine 11, eaat3005 (2019).

27. V. Schaeffer, I. Lavenir, S. Ozcelik, M. Tolnay, D. T. Winkler, M. Goedert, Stimulation of autophagy reduces neurodegeneration in a mouse model of human tauopathy. Brain 135, 2169-2177 (2012).

28. R. Siman, R. Cocca, Y. Dong, The mTOR Inhibitor Rapamycin Mitigates Perforant Pathway Neurodegeneration and Synapse Loss in a Mouse Model of Early-Stage Alzheimer-Type Tauopathy. PLoS One 10, e0142340 (2015).

29. Y. Deguchi, Application of in vivo brain microdialysis to the study of blood-brain barrier transport of drugs. Drug Metab Pharmacokinet 17, 395-407 (2002).

30. N. Strazielle, J. F. Ghersi-Egea, Potential Pathways for CNS Drug Delivery Across the BloodCerebrospinal Fluid Barrier. Curr Pharm Des 22, 5463-5476 (2016).

31. B. Birgisdottir Å, T. Johansen, Autophagy and endocytosis - interconnections and interdependencies. J Cell Sci 133, (2020).

32. L. Zeldovich, Genetic drift: the ghost in the genome. Lab Animal 46, 255-257 (2017).

33. D. R. Thal, U. Rub, M. Orantes, H. Braak, Phases of A beta-deposition in the human brain and its relevance for the development of AD. Neurology 58, 1791-1800 (2002).

34. H. Braak, E. Braak, Neuropathological stageing of Alzheimer-related changes. Acta Neuropathol 82, 239259 (1991).

35. J. B. Julian, C. F. Doeller, Remapping and realignment in the human hippocampal formation predict context-dependent spatial behavior. Nature Neuroscience, (2021).

36. L. M. Billings, S. Oddo, K. N. Green, J. L. McGaugh, F. M. LaFerla, Intraneuronal Abeta causes the onset of early Alzheimer's disease-related cognitive deficits in transgenic mice. Neuron 45, 675-688 (2005).

37. H. Zhang, W. Wei, M. Zhao, L. Ma, X. Jiang, H. Pei, Y. Cao, H. Li, Interaction between A $\beta$ and Tau in the Pathogenesis of Alzheimer's Disease. Int J Biol Sci 17, 2181-2192 (2021).

38. E. S. Blom, Y. Wang, L. Skoglund, A. C. Hansson, M. Ubaldi, A. Lourdusamy, W. H. Sommer, M. Mielke, B. T. Hyman, M. Heilig, L. Lannfelt, L. N. G. Nilsson, M. Ingelsson, Increased mRNA Levels of TCF7L2 and MYC of the Wnt Pathway in Tg-ArcSwe Mice and Alzheimer's Disease Brain. International Journal of Alzheimer\&\#x2019; s Disease 2011, 936580 (2011).

39. W. Wei, Y. Wang, J. Zhang, Q. Gu, X. Liu, L. Song, Z. Chai, M. Guo, J. Yu, C. Ma, Fasudil ameliorates cognitive deficits, oxidative stress and neuronal apoptosis via inhibiting ROCK/MAPK and activating Nrf2 signalling pathways in APP/PS1 mice. Folia Neuropathol 59, 32-49 (2021).

40. T. Hamano, N. Shirafuji, S. H. Yen, H. Yoshida, N. M. Kanaan, K. Hayashi, M. Ikawa, O. Yamamura, Y. Fujita, M. Kuriyama, Y. Nakamoto, Rho-kinase ROCK inhibitors reduce oligomeric tau protein. Neurobiol Aging 89, 41-54 (2020).

41. R. A. Nixon, D. S. Yang, Autophagy failure in Alzheimer's disease--locating the primary defect. Neurobiol Dis 43, 38-45 (2011).

42. F. M. Menzies, A. Fleming, A. Caricasole, C. F. Bento, S. P. Andrews, A. Ashkenazi, J. Füllgrabe, A. Jackson, M. Jimenez Sanchez, C. Karabiyik, F. Licitra, A. Lopez Ramirez, M. Pavel, C. Puri, M. Renna, T. Ricketts, L. Schlotawa, M. Vicinanza, H. Won, Y. Zhu, J. Skidmore, D. C. Rubinsztein, Autophagy and Neurodegeneration: Pathogenic Mechanisms and Therapeutic Opportunities. Neuron 93, 1015-1034 (2017).

43. M. R. D'Andrea, R. G. Nagele, H. Y. Wang, P. A. Peterson, D. H. Lee, Evidence that neurones accumulating amyloid can undergo lysis to form amyloid plaques in Alzheimer's disease. Histopathology 38, 120-134 (2001).

44. R. B. Knowles, C. Wyart, S. V. Buldyrev, L. Cruz, B. Urbanc, M. E. Hasselmo, H. E. Stanley, B. T. Hyman, Plaque-induced neurite abnormalities: implications for disruption of neural networks in Alzheimer's disease. Proc Natl Acad Sci U S A 96, 5274-5279 (1999).

45. M. A. DeTure, D. W. Dickson, The neuropathological diagnosis of Alzheimer's disease. Molecular Neurodegeneration 14, 32 (2019).

46. E. C. Mormino, R. A. Betensky, T. Hedden, A. P. Schultz, R. E. Amariglio, D. M. Rentz, K. A. Johnson, R. A. Sperling, Synergistic Effect of $\beta$-Amyloid and Neurodegeneration on Cognitive Decline in Clinically Normal Individuals. JAMA Neurology 71, 1379-1385 (2014).

47. C. R. Jack, Jr., H. J. Wiste, P. Vemuri, S. D. Weigand, M. L. Senjem, G. Zeng, M. A. Bernstein, J. L. Gunter, V. S. Pankratz, P. S. Aisen, M. W. Weiner, R. C. Petersen, L. M. Shaw, J. Q. Trojanowski, D. S. 
Knopman, I. the Alzheimer's Disease Neuroimaging, Brain beta-amyloid measures and magnetic resonance imaging atrophy both predict time-to-progression from mild cognitive impairment to Alzheimer's disease. Brain 133, 3336-3348 (2010).

48. R. L. Buckner, J. R. Andrews-Hanna, D. L. Schacter, The brain's default network: anatomy, function, and relevance to disease. Ann N Y Acad Sci 1124, 1-38 (2008).

49. C. R. Jack, H. J. Wiste, D. S. Knopman, P. Vemuri, M. M. Mielke, S. D. Weigand, M. L. Senjem, J. L. Gunter, V. Lowe, B. E. Gregg, V. S. Pankratz, R. C. Petersen, Rates of $\beta$-amyloid accumulation are independent of hippocampal neurodegeneration. Neurology 82, 1605 (2014).

50. R. Gail Canter, W.-C. Huang, H. Choi, J. Wang, L. Ashley Watson, C. G. Yao, F. Abdurrob, S. M. Bousleiman, J. Z. Young, D. A. Bennett, I. Delalle, K. Chung, L.-H. Tsai, 3D mapping reveals networkspecific amyloid progression and subcortical susceptibility in mice. Communications Biology 2, 360 (2019).

51. C. A. McLean, R. A. Cherny, F. W. Fraser, S. J. Fuller, M. J. Smith, K. Beyreuther, A. I. Bush, C. L. Masters, Soluble pool of Abeta amyloid as a determinant of severity of neurodegeneration in Alzheimer's disease. Ann Neurol 46, 860-866 (1999).

52. L. F. Lue, Y. M. Kuo, A. E. Roher, L. Brachova, Y. Shen, L. Sue, T. Beach, J. H. Kurth, R. E. Rydel, J. Rogers, Soluble amyloid beta peptide concentration as a predictor of synaptic change in Alzheimer's disease. Am J Pathol 155, 853-862 (1999).

53. K. M. Rodrigue, K. M. Kennedy, D. C. Park, Beta-amyloid deposition and the aging brain. Neuropsychol Rev 19, 436-450 (2009).

54. E. H. Koo, S. L. Squazzo, Evidence that production and release of amyloid beta-protein involves the endocytic pathway. J Biol Chem 269, 17386-17389 (1994).

55. A. Lorenzen, J. Samosh, K. Vandewark, P. H. Anborgh, C. Seah, A. C. Magalhaes, S. P. Cregan, S. S. G. Ferguson, S. H. Pasternak, Rapid and Direct Transport of Cell Surface APP to the Lysosome defines a novel selective pathway. Molecular Brain 3, 11 (2010).

56. A. Yu, J. F. Rual, K. Tamai, Y. Harada, M. Vidal, X. He, T. Kirchhausen, Association of Dishevelled with the clathrin AP-2 adaptor is required for Frizzled endocytosis and planar cell polarity signaling. Dev Cell 12, 129-141 (2007).

57. B. L. Heckmann, B. J. W. Teubner, B. Tummers, E. Boada-Romero, L. Harris, M. Yang, C. S. Guy, S. S. Zakharenko, D. R. Green, LC3-Associated Endocytosis Facilitates $\beta$-Amyloid Clearance and Mitigates Neurodegeneration in Murine Alzheimer's Disease. Cell 178, 536-551.e514 (2019).

58. G. M. Alexander, J. R. Grothusen, R. J. Schwartzman, Flow dependent changes in the effective surface area of microdialysis probes. Life Sci 43, 595-601 (1988).

59. H. Benveniste, Brain microdialysis. J Neurochem 52, 1667-1679 (1989).

60. C. Bjorkli, C. Louet, T. H. Flo, M. Hemler, A. Sandvig, I. Sandvig, In Vivo Microdialysis in Mice Captures Changes in Alzheimer's Disease Cerebrospinal Fluid Biomarkers Consistent with Developing Pathology. In Review J Alzheimers Dis, (2021).

61. S. Neselius, H. Brisby, A. Theodorsson, K. Blennow, H. Zetterberg, J. Marcusson, CSF-Biomarkers in Olympic Boxing: Diagnosis and Effects of Repetitive Head Trauma. PLOS ONE 7, e33606 (2012).

62. N. Marklund, K. Blennow, H. Zetterberg, E. Ronne-Engstrom, P. Enblad, L. Hillered, Monitoring of brain interstitial total tau and beta amyloid proteins by microdialysis in patients with traumatic brain injury. $J$ Neurosurg 110, 1227-1237 (2009).

63. E. Zenaro, G. Piacentino, G. Constantin, The blood-brain barrier in Alzheimer's disease. Neurobiology of disease 107, 41-56 (2017).

64. M. Ujiie, D. L. Dickstein, D. A. Carlow, W. A. Jefferies, Blood-brain barrier permeability precedes senile plaque formation in an Alzheimer disease model. Microcirculation 10, 463-470 (2003).

65. S. M. Nestor, R. Rupsingh, M. Borrie, M. Smith, V. Accomazzi, J. L. Wells, J. Fogarty, R. Bartha, t. A. s. D. N. Initiative, Ventricular enlargement as a possible measure of Alzheimer's disease progression validated using the Alzheimer's disease neuroimaging initiative database. Brain 131, 2443-2454 (2008).

66. F. A. M. Manno, A. G. Isla, S. H. C. Manno, I. Ahmed, S. H. Cheng, F. A. Barrios, C. Lau, Early Stage Alterations in White Matter and Decreased Functional Interhemispheric Hippocampal Connectivity in the 3xTg Mouse Model of Alzheimer's Disease. Front Aging Neurosci 11, (2019).

67. C. Holmes, S. Lovestone, The clinical phenotype of familial and sporadic late onset Alzheimer's disease. Int J Geriatr Psychiatry 17, 146-149 (2002). 
bioRxiv preprint doi: https://doi.org/10.1101/2022.01.14.476357; this version posted January 14, 2022. The copyright holder for this preprint

(which was not certified by peer review) is the author/funder. All rights reserved. No reuse allowed without permission.

68. F. T. Hane, M. Robinson, B. Y. Lee, O. Bai, Z. Leonenko, M. S. Albert, Recent Progress in Alzheimer's Disease Research, Part 3: Diagnosis and Treatment. Journal of Alzheimer's disease : JAD 57, 645-665 (2017).

69. J. C. Lee, S. J. Kim, S. Hong, Y. Kim, Diagnosis of Alzheimer's disease utilizing amyloid and tau as fluid biomarkers. Exp Mol Med 51, 53 (2019).

70. C. Bjorkli, Microdialysis guide cannula implantation surgery. protocols.io, (2021).

71. C. Bjorkli, M. H., Push-pull microdialysis sampling protocol. protocols.io, (2021).

72. C. Yuan, J. Gao, J. Guo, L. Bai, C. Marshall, Z. Cai, L. Wang, M. Xiao, Dimethyl sulfoxide damages mitochondrial integrity and membrane potential in cultured astrocytes. PloS one 9, e107447-e107447 (2014).

73. C. Bjorkli, IHC AD neuropathology protocol protocols.io, (2019).

74. M. R. D'Andrea, R. G. Nagele, MAP-2 immunolabeling can distinguish diffuse from dense-core amyloid plaques in brains with Alzheimer's disease. Biotech Histochem 77, 95-103 (2002).

75. S. Berg, D. Kutra, T. Kroeger, C. N. Straehle, B. X. Kausler, C. Haubold, M. Schiegg, J. Ales, T. Beier, M. Rudy, K. Eren, J. I. Cervantes, B. Xu, F. Beuttenmueller, A. Wolny, C. Zhang, U. Koethe, F. A. Hamprecht, A. Kreshuk, ilastik: interactive machine learning for (bio)image analysis. Nature Methods $\mathbf{1 6}$, 1226-1232 (2019).

76. G. Paxinos, F. K. B, The mouse brain in stereotaxic coordinates. (Gulf Professional Publishing, ed. 2nd 2004).

77. J. Cohen, A power primer. Psychol Bull 112, 155-159 (1992).

\section{SUPPLEMENTARY MATERIALS}
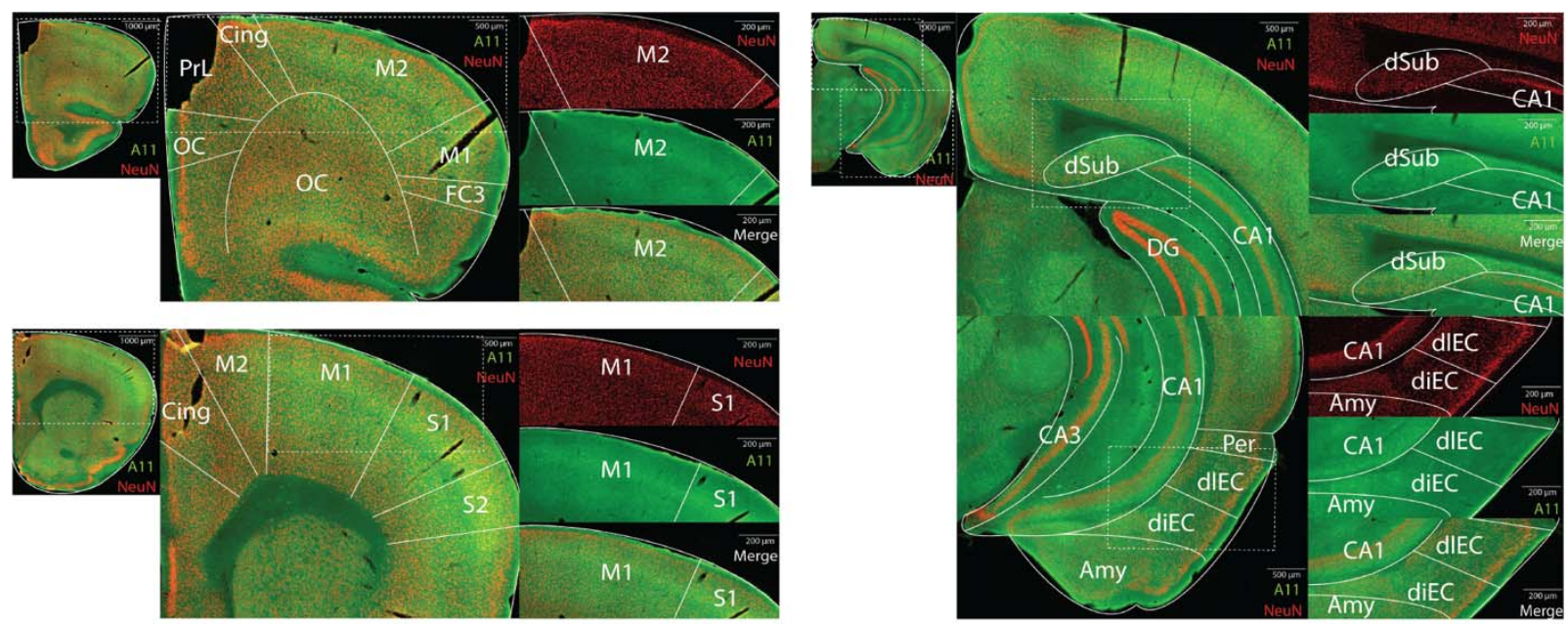

Supplementary Figure 1. Oligomeric A $\beta$ at 1-month-of-age in the 3xTg AD mouse model. A11 (oligomeric A $\beta$ specific; green) and NeuN (nuclei specific; red) immunoreactivity in the 3xTg AD mouse model at 1-month-of-age. At this age, there is little-to-none A11 immunoreactivity in frontal and sensory areas of the brain (left), as well as in the hippocampal and parahippocampal region (right). Abbreviations; S1: primary somatosensory cortex; S2: secondary somatosensory cortex; Olf: olfactory area; OC: orbital cortex; PrL: prelimbic cortex; Cing: cingulate cortex; M1: primary motor cortex; M2: secondary motor cortex; FC3: frontal cortexarea 3; Ins: insular cortex; CA1: cornu ammonis field 1; CA2: cornu ammonisfield 2; CA3: cornu ammonis field 3; Amy: amygdala; diEC: dorsal intermediate entorhinal cortex; dlEC: dorsolateral entorhinal cortex; dSub: dorsal subiculum; PER: perirhinal cortex. 


\section{Saline}

McSA1

$200 \mu \mathrm{m}$

831 Supplementary Figure 2. Intracellular A $\beta$ in dSub after Lonafarnib infusions.

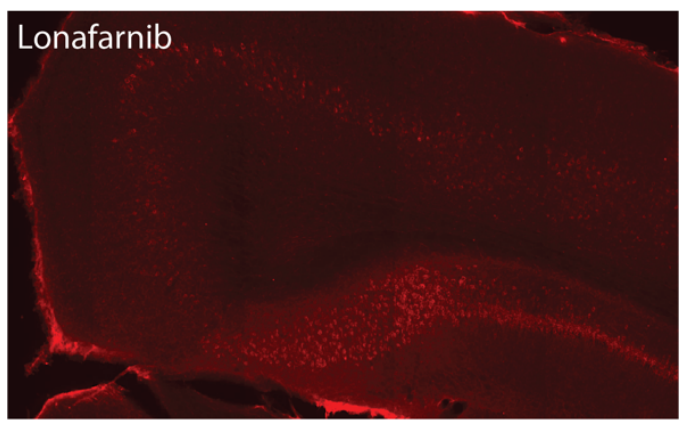

832

\section{Drug treatment day 10}

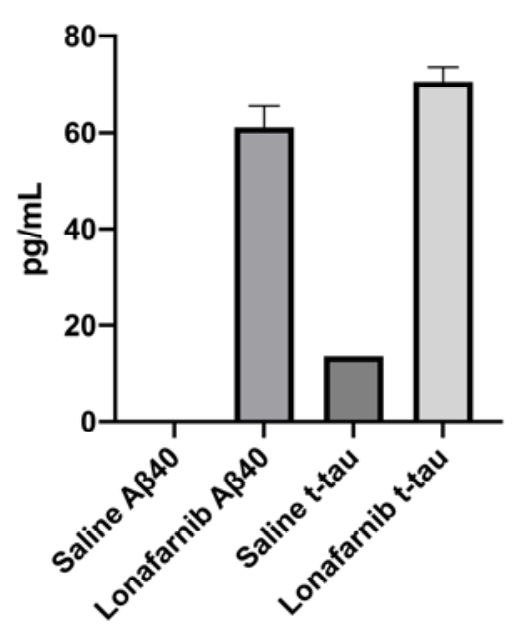

Supplementary Figure 3. CSF Aß40 and t-tau levels after Lonafarnib infusions in old mice. Drug treatment day $10, \mathrm{~N}=4$. 

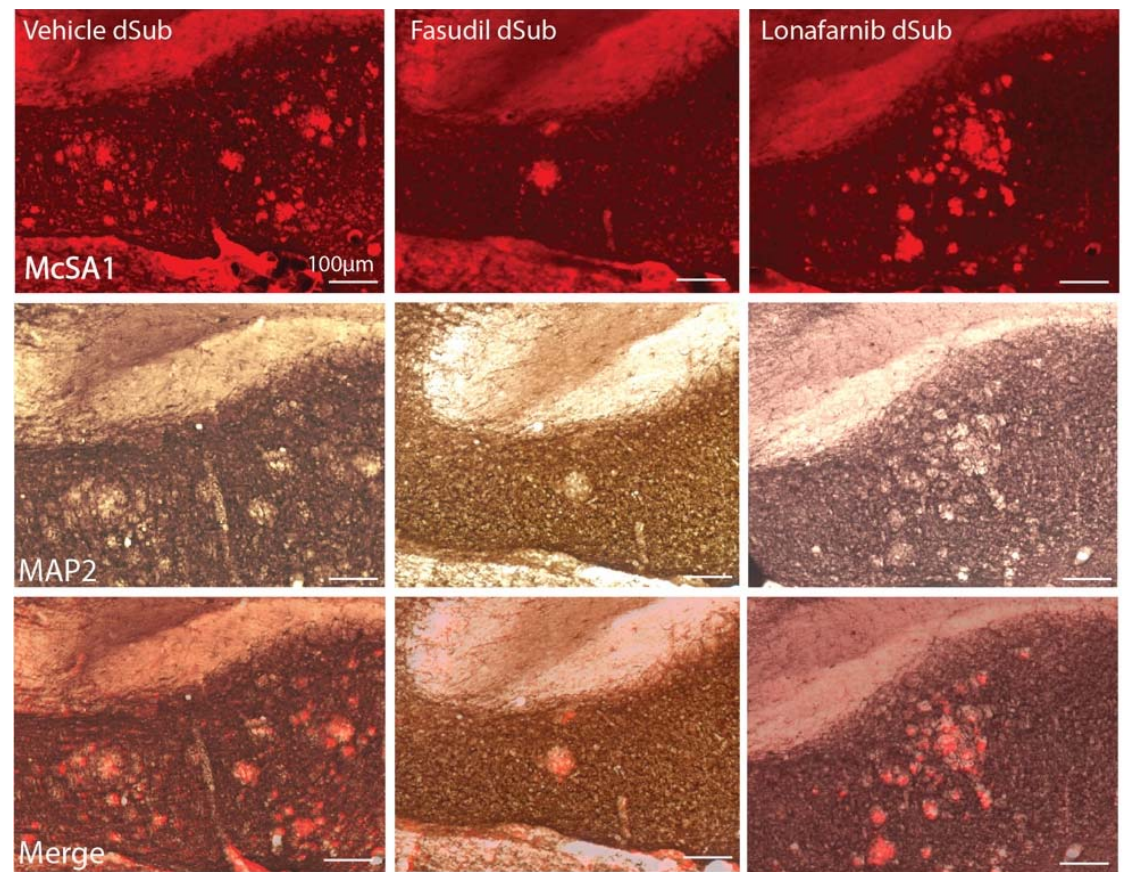

838 Supplementary Figure 4. Dense-core amyloid plaques after Fasudil and Lonafarnib infusions.

839
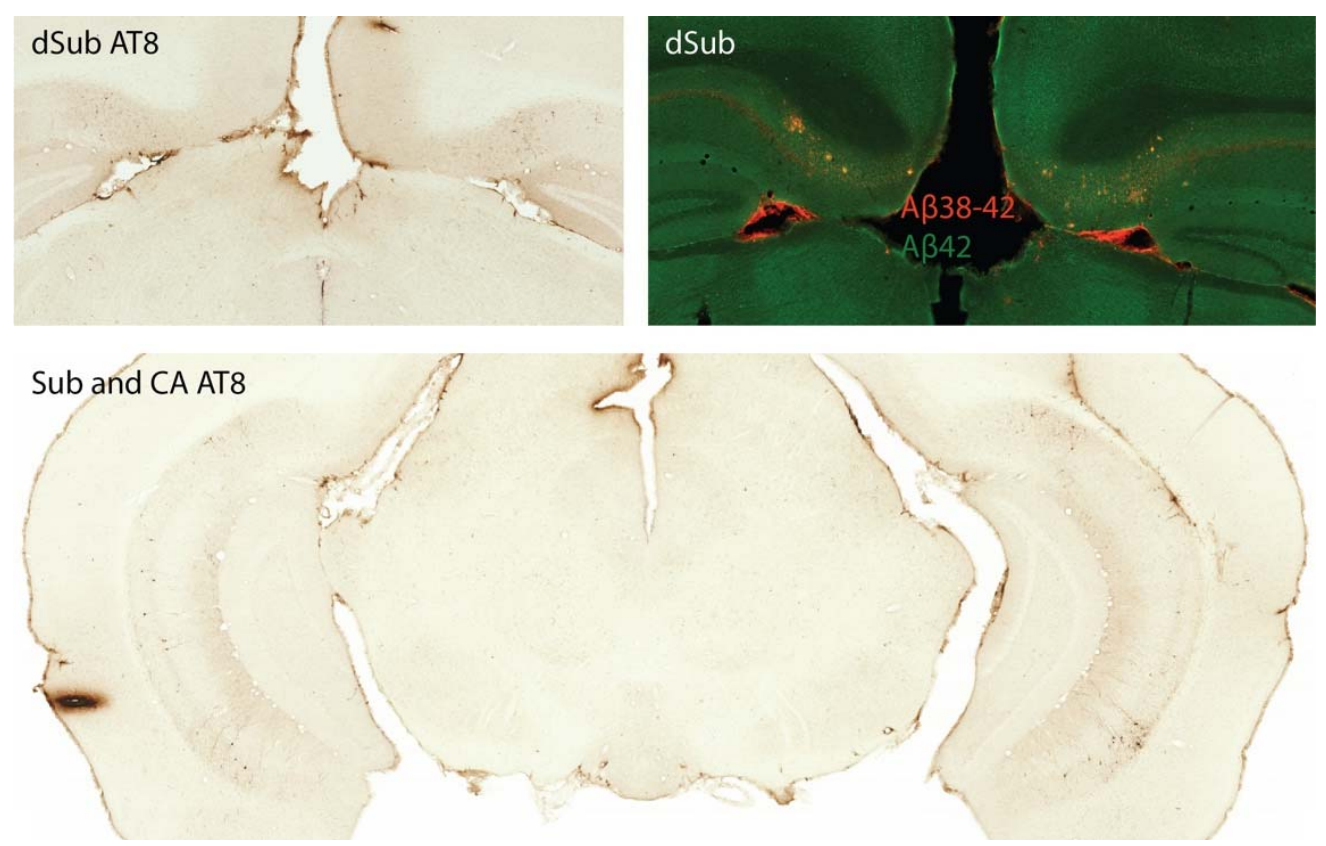

841 Supplementary Figure 5. No lateralization of pathology in 3xTg AD mice. 


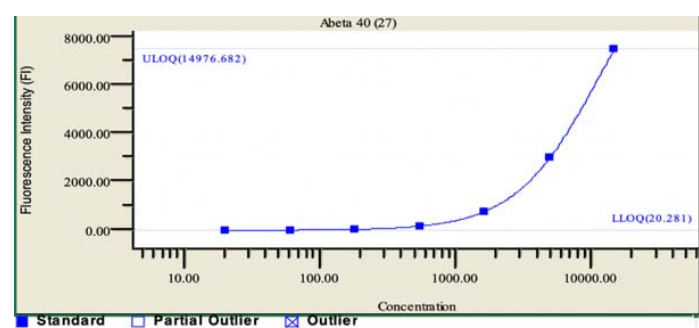

Regression Type: Logistic - 5PL

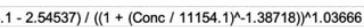
FitProb. $=0.6508$, ResVar. $=0.4296$

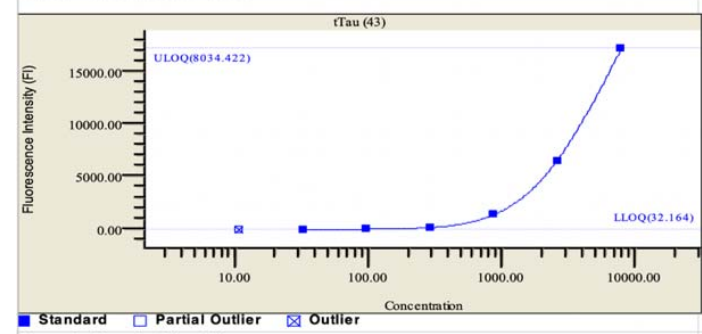

Regression Type: Logistic - 5PL

$154933) /\left(\left(1+(\text { Conc } / 2728.47)^{\mu}-0.942514\right)\right)^{2}-41084$

Supplementary Figure 6. LLQQ levels of CSF proteins.

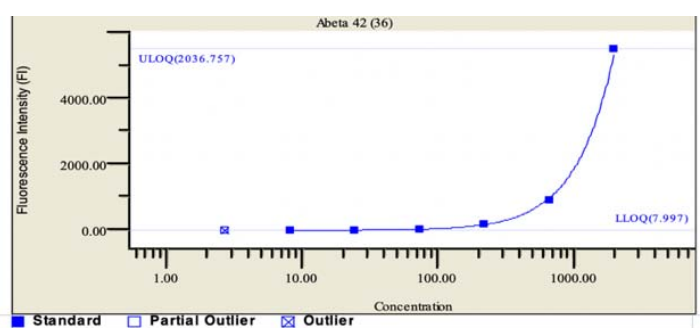

Regression Type: Logistic - 5PL

$+(5.52748 \mathrm{E}+007-0.178659) /\left(\left(1+(\mathrm{Conc} / 563008)^{\mu}-0.54329\right)\right)^{\wedge} 2.97115$ FitProb. $=0.1208$, ResVar. $=2.4064$

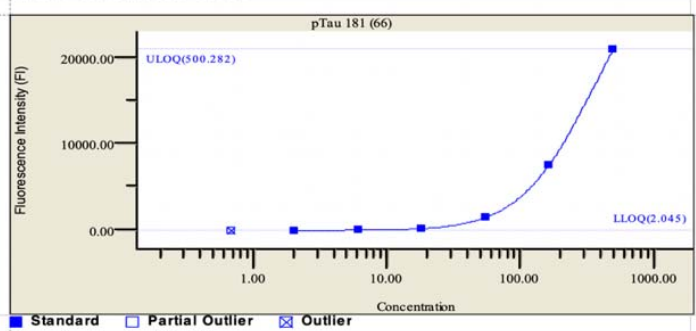

- Standard $\square$ Partial Outlier $\otimes$ Outlier

Regression Type: Logistic - 5PL

. 3.40401)/((1+ (Conc/ 280.285) -1.32071$))^{M} .41786$

ResVar $=0.045$

\section{6}

\section{Supplementary Table 1: List of antibodies used}

\begin{tabular}{|l|l|l|}
\hline Antibody & Target & Identifier \\
\hline Mouse anti-A $\beta(\mathrm{McSA} 1)$ & $\mathrm{A} \beta 38-42$ & MM-0015-1P \\
\hline Rabbit anti-human A $\beta$ & $\mathrm{A} \beta 42$ & JP28051 \\
\hline Mouse anti-Iba1 & $\begin{array}{l}\text { Ionized calcium binding } \\
\text { adaptor molecule 1 } \\
\text { (Iba1) }\end{array}$ & ab15690 \\
\hline Rabbit anti-oligomer (A11) & $\mathrm{A} \beta 42$ oligomers & AHB0052 \\
\hline $\begin{array}{l}\text { Rabbit anti-amyloid fibrils } \\
\text { (OC) }\end{array}$ & $\begin{array}{l}\text { Generic epitopes common to } \\
\text { amyloid fibrils and fibrillar } \\
\text { oligomers }\end{array}$ & AB2286 \\
\hline Mouse anti-MC1 & $\begin{array}{l}\text { AD-specific conformational } \\
\text { modification of tau }\end{array}$ & $\begin{array}{l}\text { Peter Davies, } \\
\text { Department of } \\
\text { Pathology, Albert } \\
\text { Einstein College of } \\
\text { Medicine }\end{array}$ \\
\hline Rabbit anti-TREM2 & TREM2 receptor & MA530971 \\
\hline Rabbit anti-MAP2 & $\begin{array}{l}\text { Microtubule-associated } \\
\text { protein 2 }\end{array}$ & ab183830 \\
\hline Rabbit anti-LAMP1 & $\begin{array}{l}\text { Lysosomal associated } \\
\text { membrane protein 1 }\end{array}$ & L1418 \\
\hline AT8 & $\begin{array}{l}\text { Tau phosphorylated at serine } \\
202 \text { and threonine 205 }\end{array}$ & MN1000 \\
\hline Mouse anti-HT7 & MAPT & MN1000 \\
\hline
\end{tabular}

